\title{
Addressing the physician burnout epidemic with resilience curricula in medical education: a systematic review
}

\author{
Chanhee Seo ${ }^{1 \dagger}$, Mario Corrado ${ }^{1 \dagger}$, Karine Fournier ${ }^{2}$, Tayler Bailey ${ }^{3}$ and Kay-Anne Haykal ${ }^{1 *}$
}

\begin{abstract}
Background: A variety of stressors throughout medical education have contributed to a burnout epidemic at both the undergraduate medical education (UGME) and postgraduate medical education (PGME) levels. In response, UGME and PGME programs have recently begun to explore resilience-based interventions. As these interventions are in their infancy, little is known about their efficacy in promoting trainee resilience. This systematic review aims to synthesize the available research evidence on the efficacy of resilience curricula in UGME and PGME.
\end{abstract}

Methods: We performed a comprehensive search of the literature using MEDLINE, EMBASE, PsycINFO, Educational Resources Information Centre (ERIC), and Education Source from their inception to June 2020. Studies reporting the effect of resilience curricula in UGME and PGME settings were included. A qualitative analysis of the available studies was conducted in accordance with the Preferred Reporting Items for Systematic Reviews and Meta-Analyses (PRISMA) guidelines. Risk of bias was assessed using the ROBINS-I Tool.

Results: Twenty-one studies met the inclusion criteria. Thirteen were single-arm studies, 6 quasi-experiments, and 2 RCTs. Thirty-eight percent $(8 / 21 ; n=598)$ were implemented in UGME, while $62 \%(13 / 21, n=778)$ were in PGME.

There was significant heterogeneity in the duration, delivery, and curricular topics and only two studies implemented the same training model. Similarly, there was considerable variation in curricula outcome measures, with the majority reporting modest improvement in resilience, while three studies reported worsening of resilience upon completion of training. Overall assessment of risk of bias was moderate and only few curricula were previously validated by other research groups.

Conclusions: Findings suggest that resilience curricula may be of benefit to medical trainees. Resilience training is an emerging area of medical education that merits further investigation. Additional research is needed to construct optimal methods to foster resilience in medical education.

Keywords: Systematic review, Resilience, Curriculum, Undergraduate medical education (UGME), Post-graduate medical education (PGME)

\footnotetext{
*Correspondence: Khaykal@uottawa.ca

${ }^{\dagger}$ Chanhee Seo and Mario Corrado contributed equally to this work.

'Department of Family Medicine, Faculty of Medicine, University of Ottawa,

451 Ch. Smyth Rd. (2024), Ottawa, ON K1H 8M5, Canada

Full list of author information is available at the end of the article
}

(C) The Author(s). 2021 Open Access This article is licensed under a Creative Commons Attribution 4.0 International License, which permits use, sharing, adaptation, distribution and reproduction in any medium or format, as long as you give appropriate credit to the original author(s) and the source, provide a link to the Creative Commons licence, and indicate if changes were made. The images or other third party material in this article are included in the article's Creative Commons licence, unless indicated otherwise in a credit line to the material. If material is not included in the article's Creative Commons licence and your intended use is not permitted by statutory regulation or exceeds the permitted use, you will need to obtain permission directly from the copyright holder. To view a copy of this licence, visit http://creativecommons.org/licenses/by/4.0/ The Creative Commons Public Domain Dedication waiver (http://creativecommons.org/publicdomain/zero/1.0/) applies to the data made available in this article, unless otherwise stated in a credit line to the data. 


\section{Background}

Medical studies present a unique set of challenges and stressors that reach beyond the academic curriculum. In addition to a substantial workload, medical students and residents are confronted with a wide range of stressors including inflexible work schedules, sleep deprivation, fatigue, time-consuming clerical and administrative responsibilities, insufficient access to allied health personnel and staff (e.g., nurses, social workers), and unwelcoming learning environments [1]. Coupled with societal- and selfexpectations [2], medical students and residents are at high risk for compassion fatigue [3-5], low self-esteem [6], decreased mental health [7-13], and overall poor quality of life [14-20]. The implications of elevated psychological distress among medical students and residents are welldocumented and include diminished academic accomplishment [21, 22], substance abuse [9, 23-25], decreased empathy [26, 27], increased professional misconduct [28], and suicide $[29,30]$. An abundance of systematic reviews have highlighted the significantly negative impact of medical education on student wellness [31-38]. Specifically, a worrying prevalence of burnout has been widely reported among medical students and residents across several specialties and numerous countries including the United States, Qatar, Australia, Canada, New Zealand, France, England, Switzerland, Thailand, India, Germany, Greece, and Trinidad \& Tobago [9, 10, 30, 39-47]. Burnout is traditionally defined as a maladaptive response to chronic work stress $[48,49]$ and characterized by a triad of emotional exhaustion (i.e., loss of enthusiasm), depersonalization (i.e., cynical thought patterns) and a reduced sense of personal accomplishment. Together, they work to negatively impact the well-being of both the medical student and the patient population [50].

The burnout epidemic among medical trainees was only recently acknowledged. In the 1990s, the "Triple Aim" of healthcare was established by healthcare institutions and organizations in the US. This new initiative emphasized patient satisfaction, quality of care and cost reductions [51]. It was not until the 2000s that staff and healthcare provider satisfaction was formally considered in the rebranded "Quadruple Aim" of healthcare [52]. In 2015, physician groups in both the United States and Canada began to adopt formal campaigns recognizing the resident burnout epidemic. In the United States, the Accreditation Council for Graduate Medical Education (ACGME) amended its accreditation requirements to address trainee well-being and resilience more comprehensively across all residency and fellowship programs [53]. In addition to providing self-care resources, the campaign helped residents find meaning in their work, enhanced communication and professional relationships, evaluated and promoted safety in the working and learning environment, and provided education and resources to identify and treat burnout, depression, substance abuse and other challenges [54]. In Canada, the 2015 CanMEDS Physician Competency Framework outlined that, in order to be a professional, one must have a commitment to self [55]. In response, an increasing number of residency programs have restructured their training to provide and foster specific skills and dispositions towards work-life balance and self-care $[56,57]$.

The ACGME's formal recognition of the resident burnout epidemic paved the way for new wellness initiatives. Institutions have begun exploring a vast array of healthpromotion programs including mindfulness [58-60], yoga [61, 62], self-hypnosis protocols [63], small group debriefing and stress-management programs [64], curricular changes [65], evaluation changes [66, 67], time management programs [68], reflective writing sessions $[69,70]$, and self-development groups [71]. However, recent literature on therapeutic stress management programs for medical students and residents have revealed mixed and inconsistent results, with overall unclear long-term benefits. In response, medical schools and residency programs have begun exploring alternate wellness interventions, aiming to prevent burnout long-term by fostering trainee resilience [72-74].

Resilience is broadly defined but can be conceptualized as the ability to face adversity forthrightly and intentionally instead of aiming merely to survive through hardships. Resilient individuals re-frame challenges as opportunities for growth and thus willingly engage with the harsh realities of life in a healthy manner that ultimately achieves goals at a minimal physical and psychological cost [75]. A systematic review on resilience identified five themes used to define resilient individuals: rising above adversity, adapting and adjusting, resilience as a dynamic process, "ordinary magic" (i.e., resilience is an inherent trait in all people) and mental illness as a marker of resilience [76]. Resilience was initially regarded as an inherited, static character trait [77, 78], however research has identified it as a dynamic and transient quality [78]. Importantly, resilience education requires a foundation of self-awareness and the ability to self-monitor [75]. This necessitates that the individual willingly accepts their limits and uncertainties, and uses their insight to recognize errors and problem solve [77]. Initial studies have indicated that physicians who exhibit high-resilience personality traits have an objectivelyelevated sense of overall well-being, provide better quality patient care and ultimately contribute to an overall decrease in healthcare costs [79].

These initial observations support the implementation of resilience-based interventions in medical training to prepare trainees for the inevitable hardships of clinical practice $[75,80]$. Although such interventions have been developed and integrated into medical school and 
residency programs, little is known about their efficacy in promoting resilience. To address this paucity in the literature, we conducted a systematic review to synthesize the available research evidence on the efficacy of resilience curricula in undergraduate and postgraduate medical education.

\section{Methods}

\section{Protocol and registration}

A systematic review protocol was prospectively developed and registered in the International Prospective Register of Systematic Reviews (PROSPERO, CRD42020191511). This systematic review was performed in concordance with the Cochrane Handbook for systematic reviews of interventions and the Preferred Reporting Items for Systematic Reviews and Meta-Analysis Protocols (PRISMA-P) statement (Additional file 1) [81-83].

\section{Search method}

A systematic search of the literature was performed by an information specialist (K.F.) in MEDLINE(R) ALL (Ovid, 1946 to June 15, 2020), Embase (Ovid, 1947 to 2020 June 15), APA PsycInfo (Ovid, 1806 to June Week 22,020), ERIC (Ovid, 1965 to March 2020), Education Source (EBSCOHost, 1880-2020) from database inception to June 16, 2020 using a combination of subject headings terms and keywords (Additional file 2): "medical education", "medical student", "resilience", "curriculum", and "training". In addition, a manual search of the reference lists of the retrieved articles was conducted to capture all relevant studies for potential inclusion.

\section{Eligibility criteria}

We included all peer reviewed primary research articles that implemented resilience curricula in UGME and PGME settings and reported efficacy outcome measures. As per our review protocol published a priori, the inclusion criteria for studies were: (1) both single- and double-arm studies of either qualitative or quantitative nature in which the participants received a clearly defined curriculum which included at least one concept of resilience including, but not limited to, coping skills, self-efficacy, goal-setting, and emotional regulation skills; (2) study participants including undergraduate and postgraduate medical learners; (3) an outcome measure assessing the effect of resilience curricula through quantitative or qualitative research instruments. Both randomized controlled trials and quasi-experimental studies were included. No restrictions were placed on the study subjects' age, gender, country, level or specialty of training, and length of curricula and follow-up period.

We excluded review articles, editorials, letters, commentaries, theoretical articles (e.g., curricular development), and non-English articles; however, a manual search of the reference lists of review articles was conducted to ensure broad and comprehensive inclusion of the available literature. For the purpose of this review, studies were excluded if their primary focus was therapeutic in nature (e.g., cognitive therapy, counselling, and mind-body skills). Finally, studies that only described participant satisfaction or those that only described the implementation of resilience curricula were also excluded.

\section{Study selection and data abstraction}

After identifying all citations and removing duplicates, a two-step screening process was employed to determine the relevancy of the identified articles. First, each study title and abstract was independently screened for eligibility in accordance with the aforementioned eligibility criteria by two reviewers (C.S. and M.C.). An inter-rater calibration test was performed using 20 randomly identified articles prior to formally commencing the screening process to ensure reliable screening accuracy. Following the title-abstract screening, the same two reviewers independently retrieved the full text of relevant articles and determined their eligibility for inclusion using the preset inclusion criteria. Any discrepancies between the two reviewers were resolved by consensus. When no consensus could be reached, a third reviewer was consulted.

A standardized data collection table was created by the review team prior to formally commencing the extraction process. Extracted variables included: (1) study characteristics (e.g., country of origin, study design, target population, sample size); (2) curriculum description (e.g., name, curricular content, delivery mode, duration and frequency); (3) resilience outcome scales; and (4) secondary curriculum outcome measures. Two reviewers (C.S. and M.C.) independently extracted the data and compared the results for verification. Any disagreements between the two reviewers were resolved by consensus after consulting the entire review team.

\section{Analysis of studies}

A primarily qualitative appraisal of the literature was conducted following the methodological guidelines for qualitative reviews outlined in the Cochrane Handbook [83]. When appropriate, descriptive statistics were utilized to present the overall trend or effect of results. Due to the heterogeneity in both curricula and outcome measures across the included studies, a pooled analysis of the effects of curricula using a meta-analytic methodology was not possible.

\section{Quality appraisal}

A risk of bias assessment of each included study was performed by two independent reviewers (C.S. and M.C.) using the ROBINS-I tool $[84,85]$. Risk of bias was 
assessed across seven domains (confounding, selection, classification, intervention, missing data, measurement, reporting). Results of quality assessments were compared, and when there were any disagreements, the entire review team was consulted which were then resolved by consensus.

\section{Results}

\section{Study selection}

Figure 1 depicts the study selection process in a PRIS MA flow diagram. The initial electronic search of the databases identified 6101 citations. Two thousand one hundred one duplicate records were removed using Covidence (Veritas Health Information, Melbourne, Australia), which left 4000 references for the screening phase. Following the title-abstract screening, 3886 studies were deemed to be irrelevant for the present review based on the exclusion criteria. Inter-rater reliability for the title-abstract screening was excellent $(k>0.90)$. Of the 114 studies assessed for eligibility in the full-text screening, 93 studies were further excluded as they did not meet the inclusion criteria. No additional studies were identified after a manual screening of reference lists of the included studies. In total, 21 studies that fully satisfied the previously stipulated eligibility criteria were included in this review [57, 86-105].

\section{Study characteristics}

The characteristics of the included studies are collated and summarized in Table 1 . Of the 21 studies included in this review, 13 studies (62\%, $n=984$ total participants) used a single-arm design that evaluated the effect of resilience curricula pre- and post-intervention, 2 studies $(9.5 \%, n=100)$ used a randomized controlled design, and $6(28.5 \%, n=292)$ used a quasi-experimental design. Sample sizes of studies varied between 12 and 258, but none of the studies performed an a priori sample size determination. As shown in Fig. 2, the earliest study on resilience curricula in medical education was published in 1982 , with the majority of studies $(90.5 \%, 19 / 21)$ published in the last decade.

\section{Study populations}

Thirty-eight percent $(8 / 21, n=598$ total participants) of the included studies implemented their resilience curricula in a UGME setting: 3 studies were implemented in pre-clerkship, 2 in clerkship, 2 in both pre-clerkship and clerkship, and 1 study did not specify the level of UGME

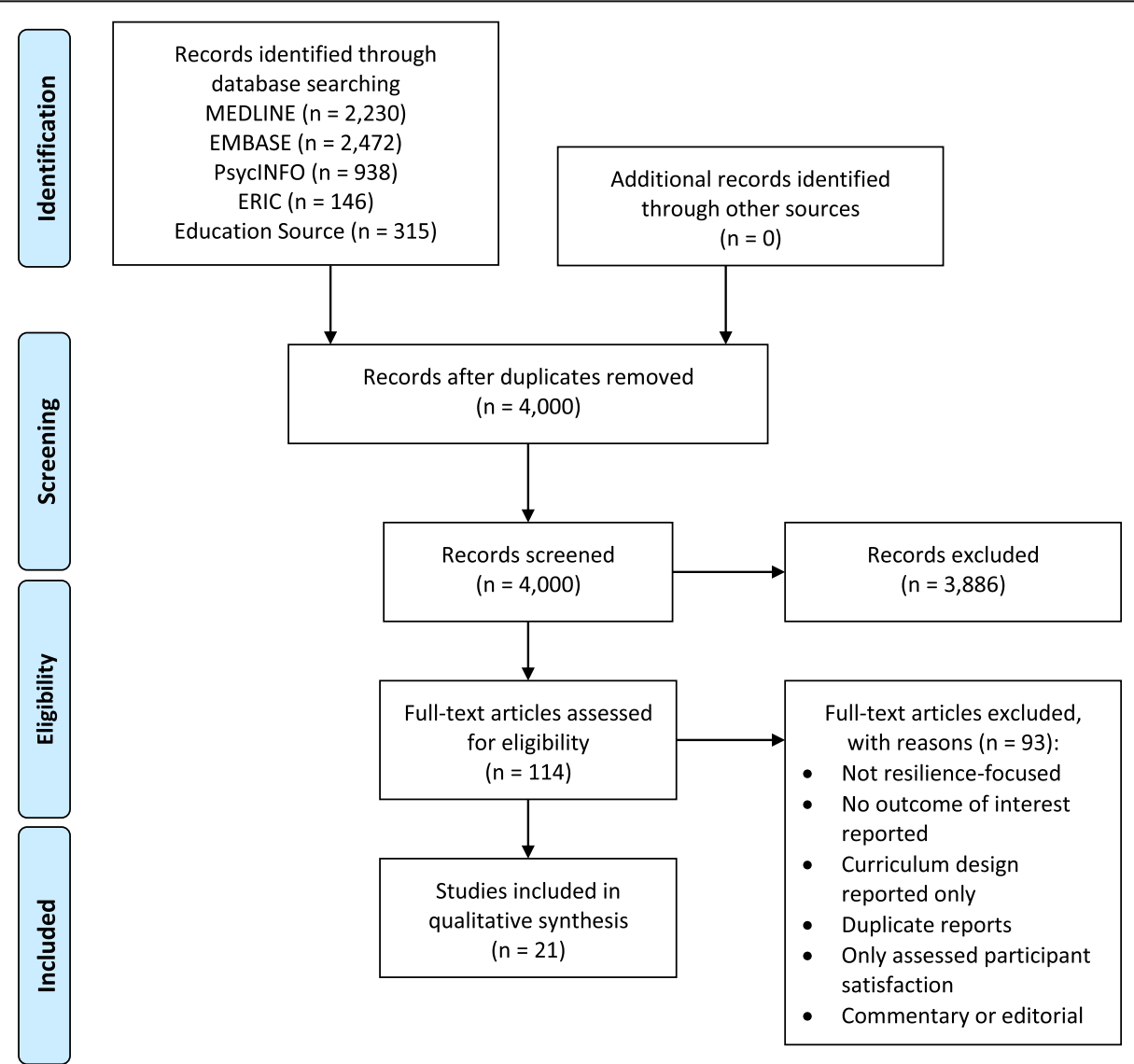

Fig. 1 PRISMA flow diagram of the study screening process 


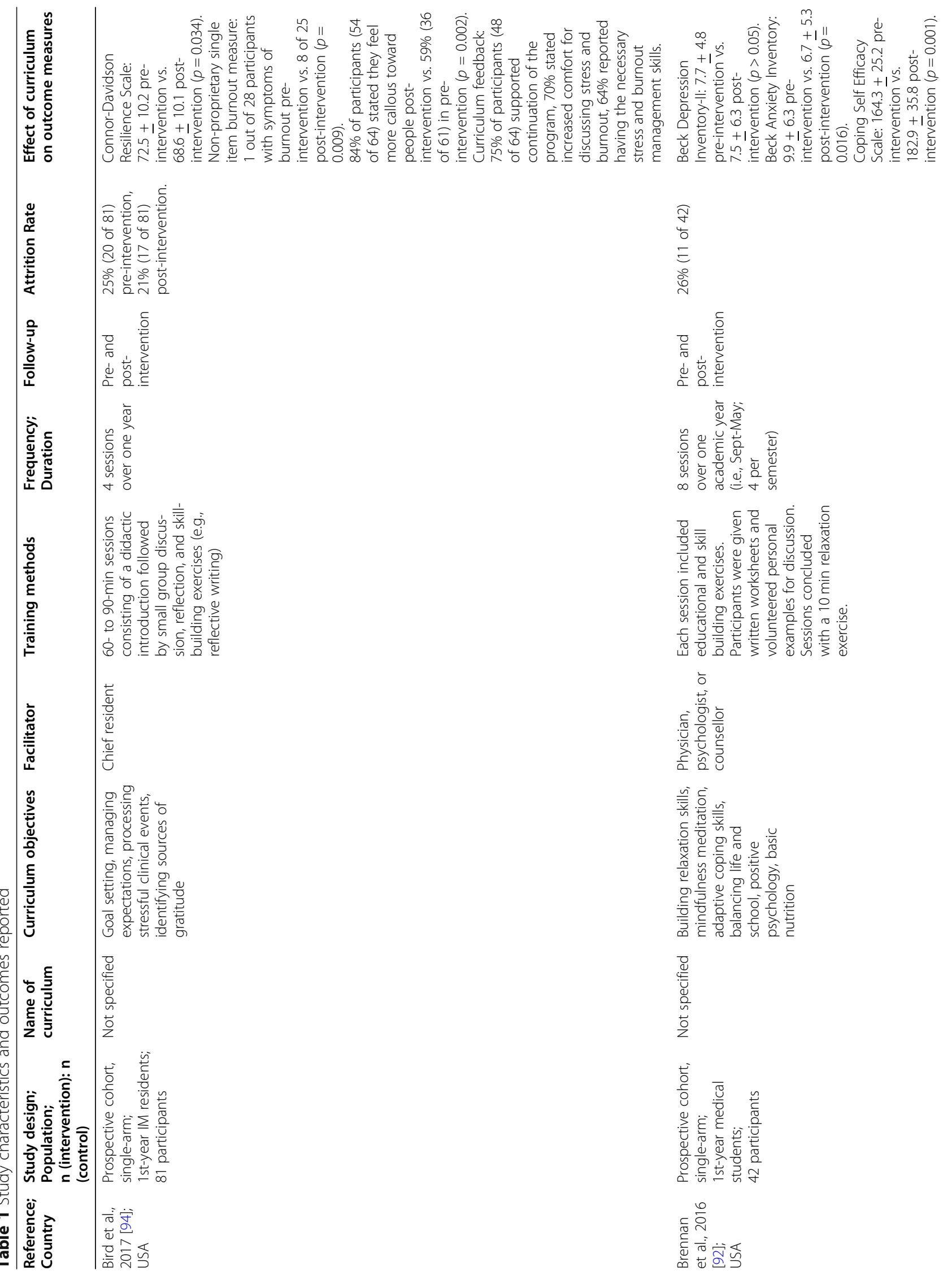




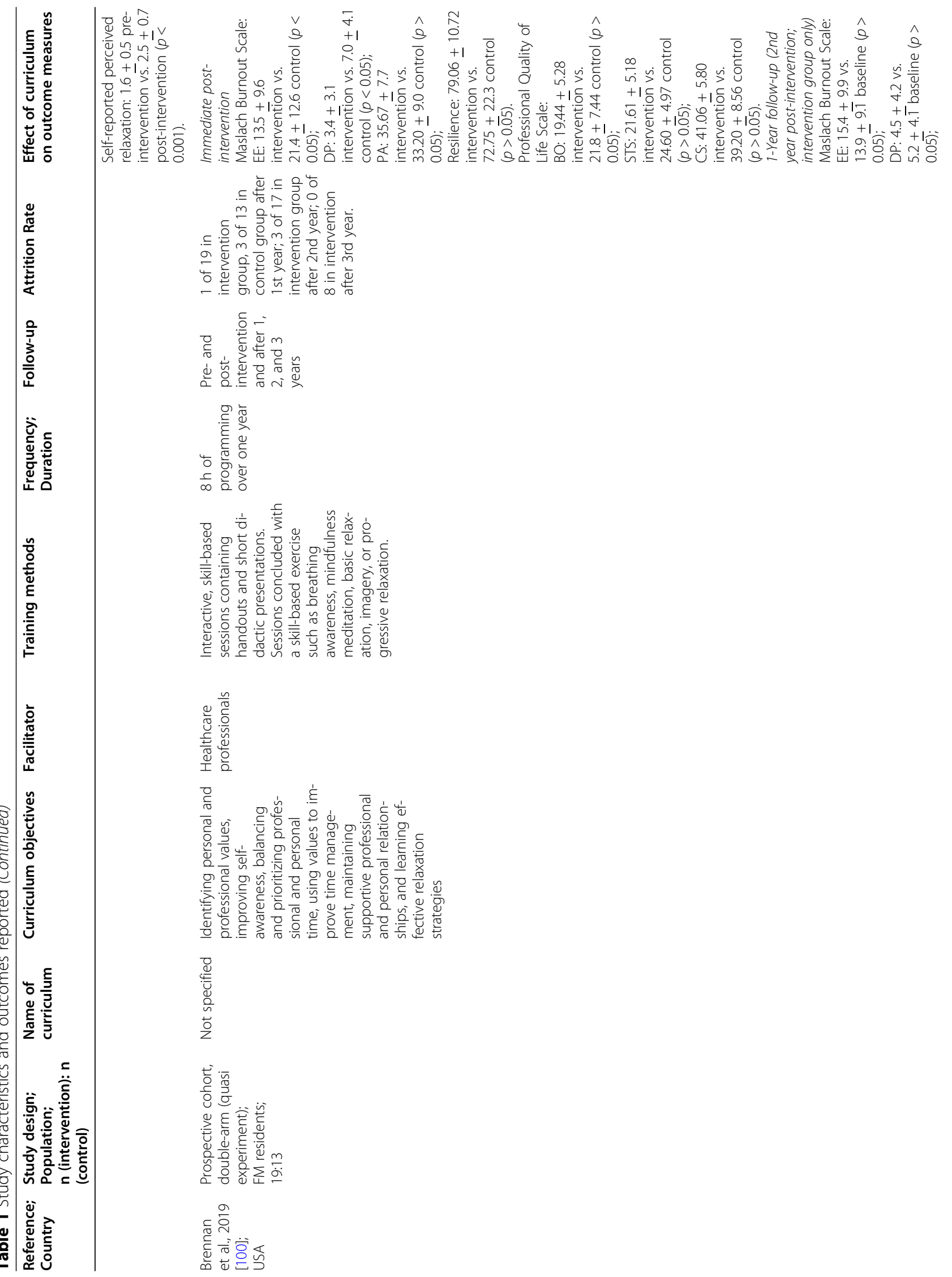




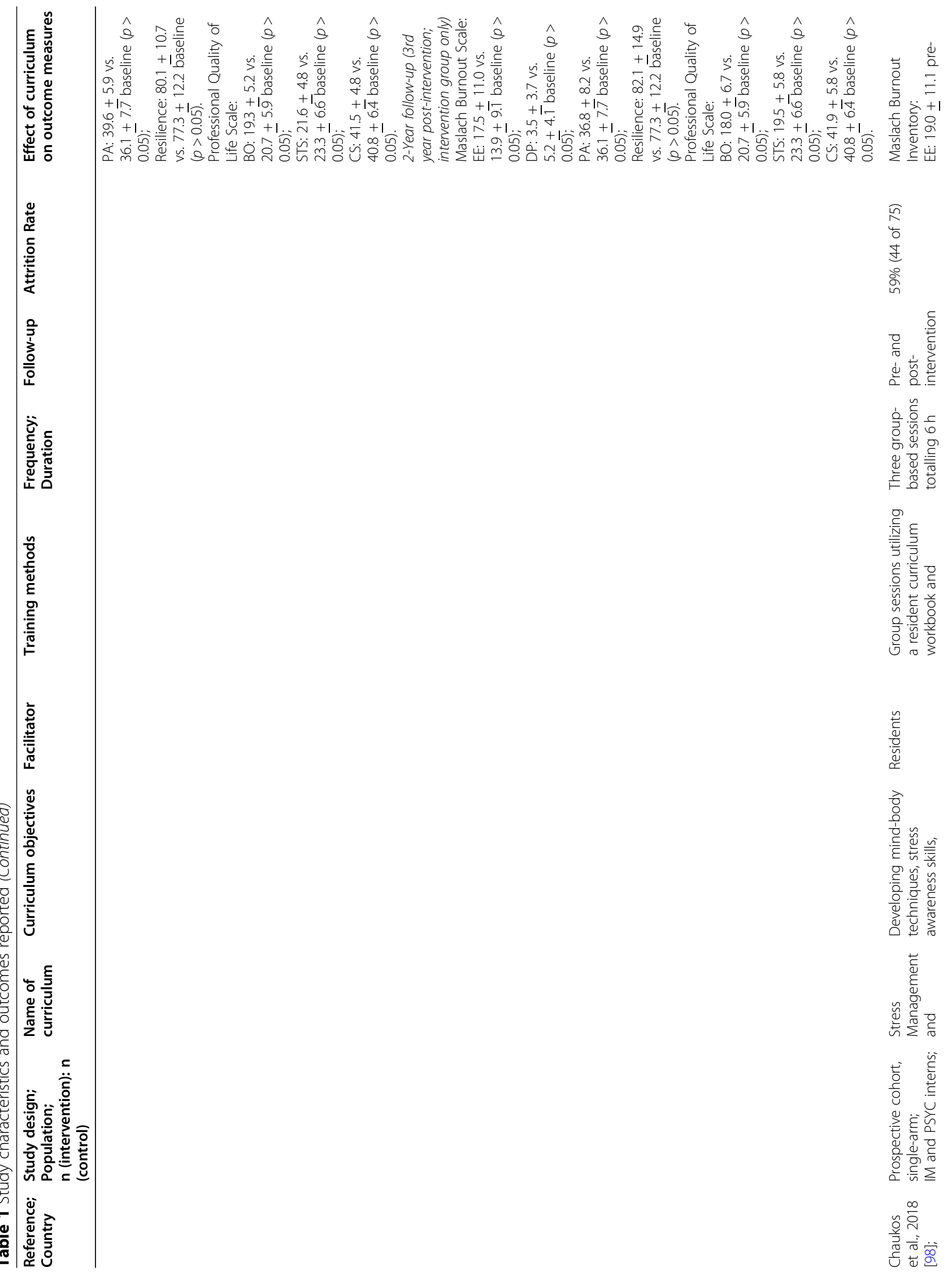




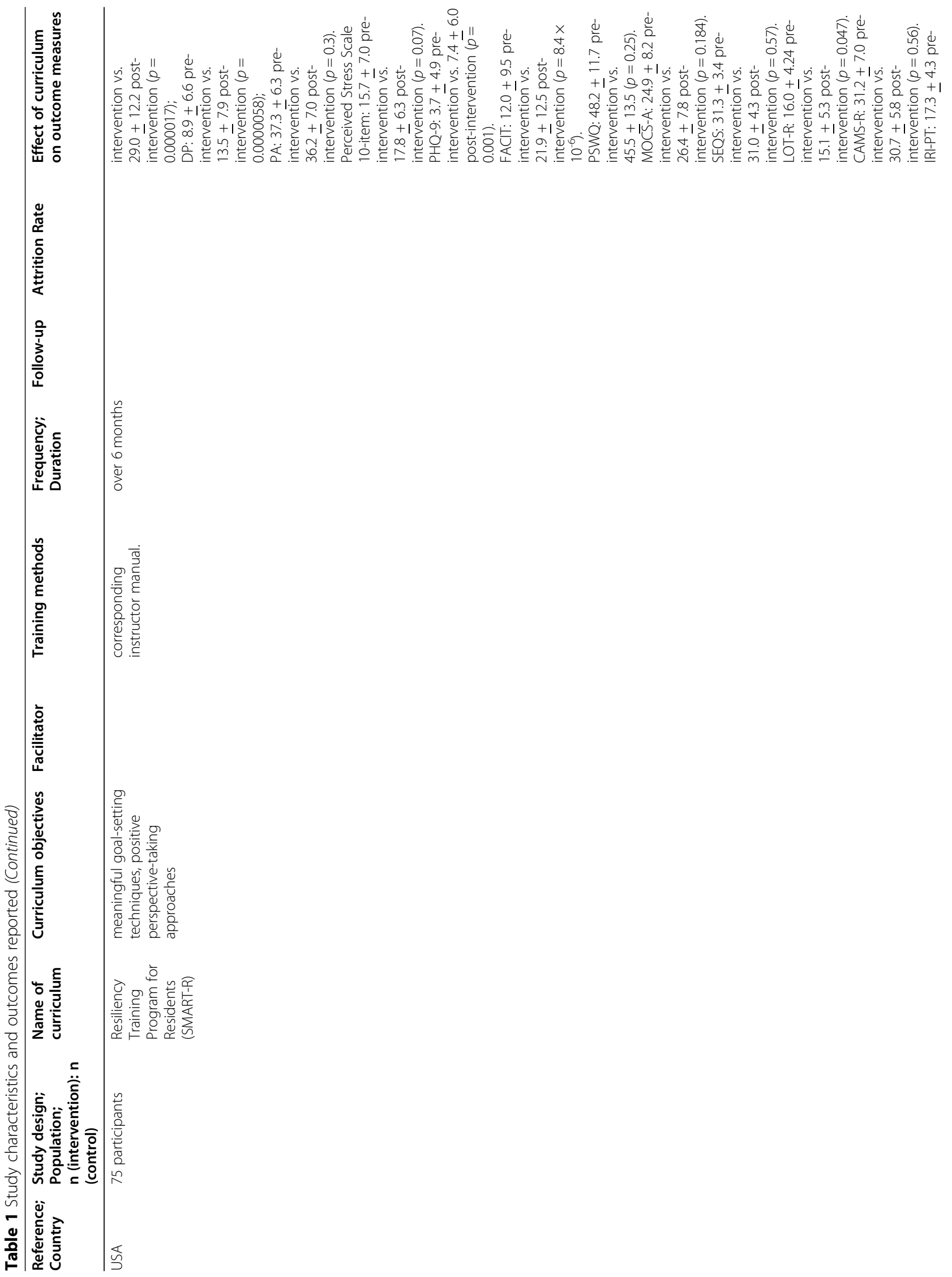




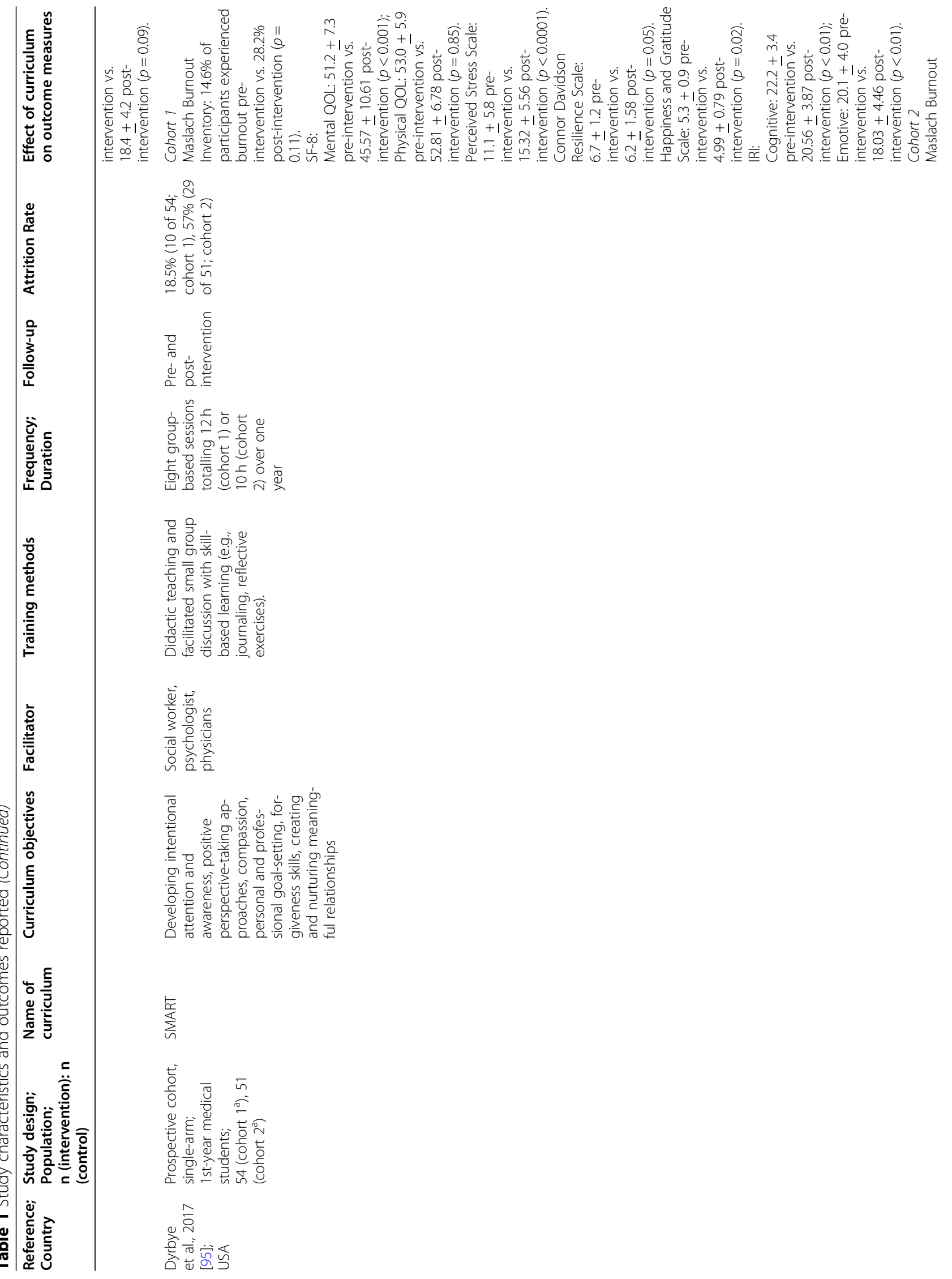




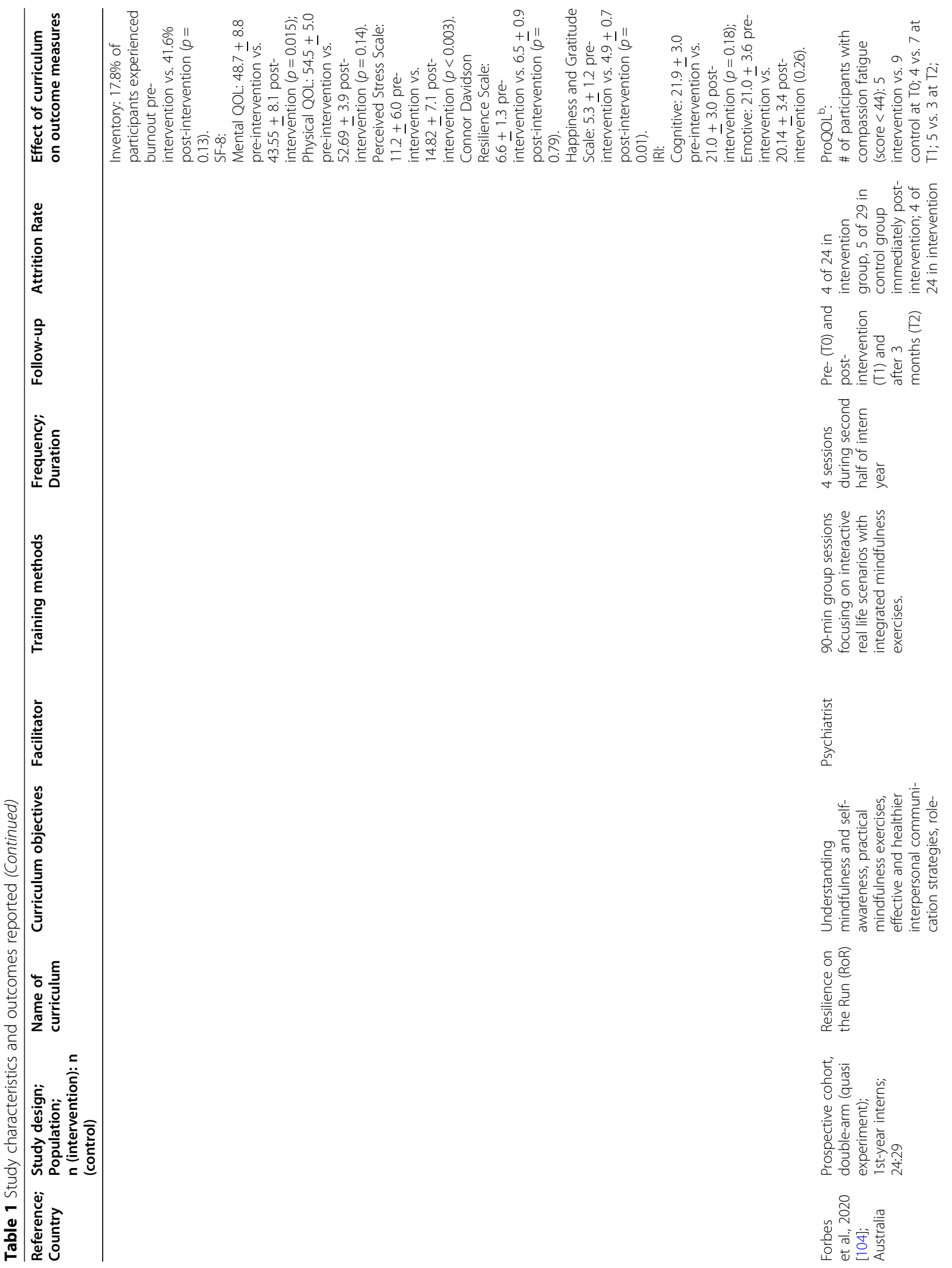




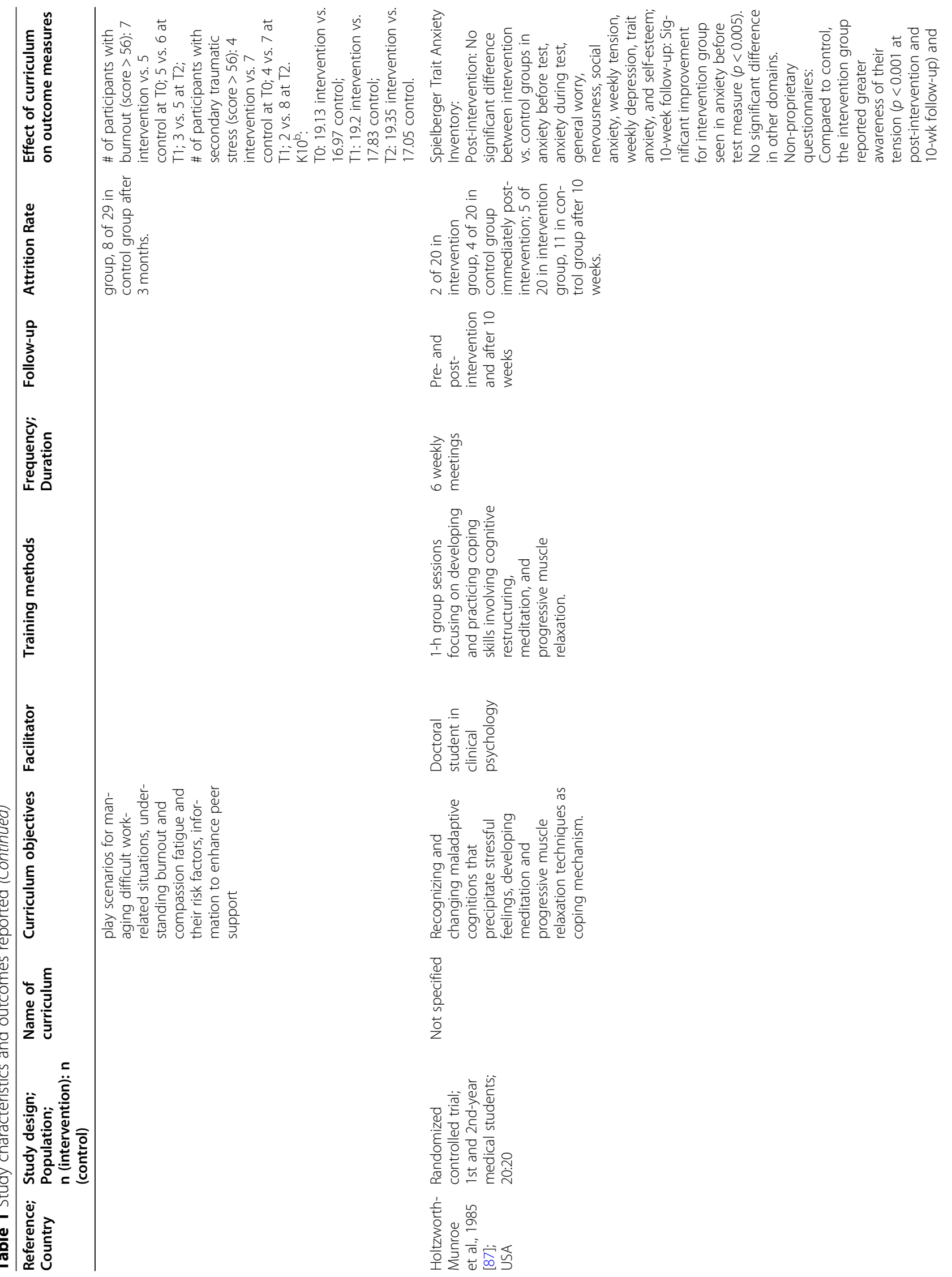




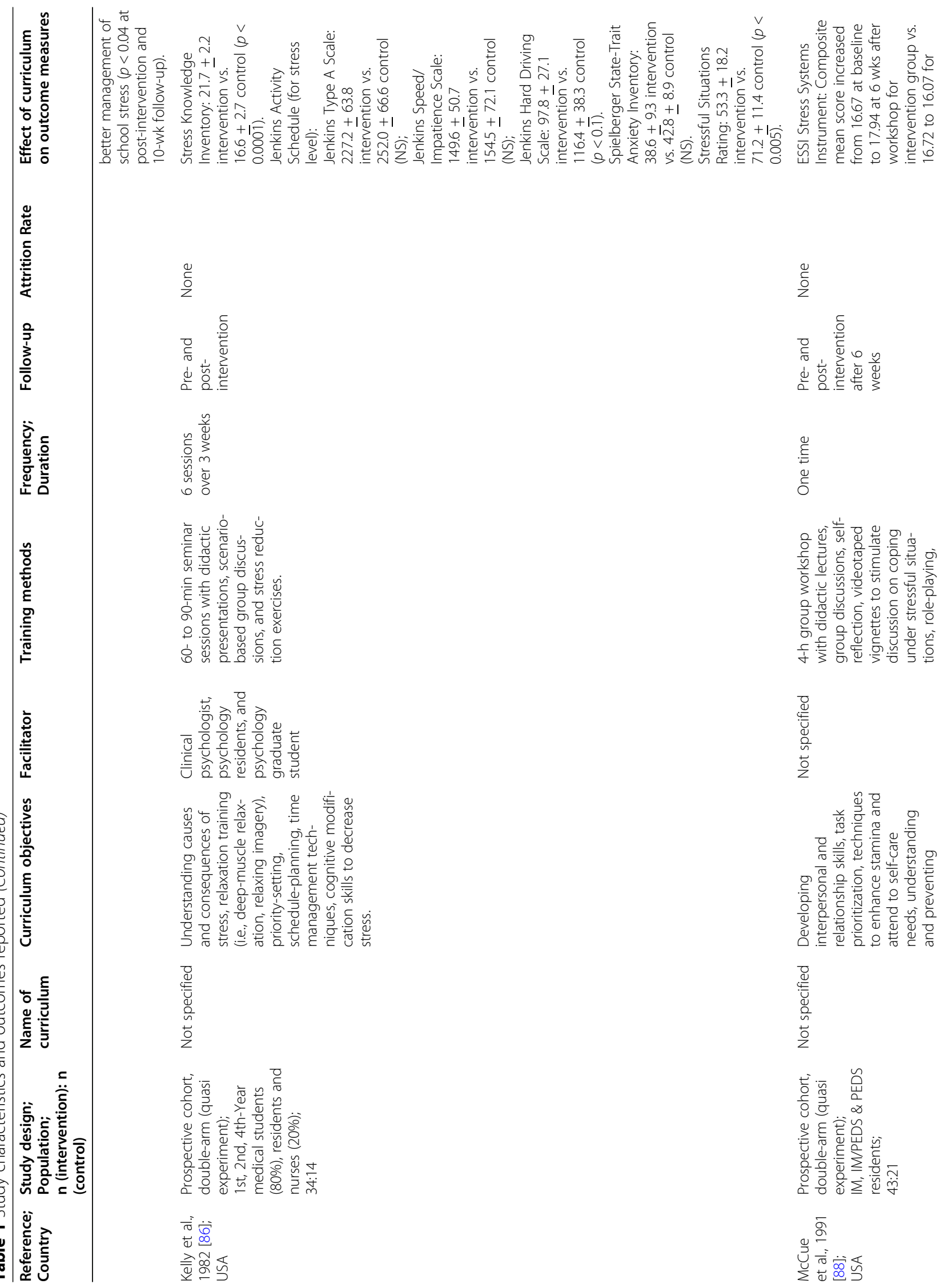




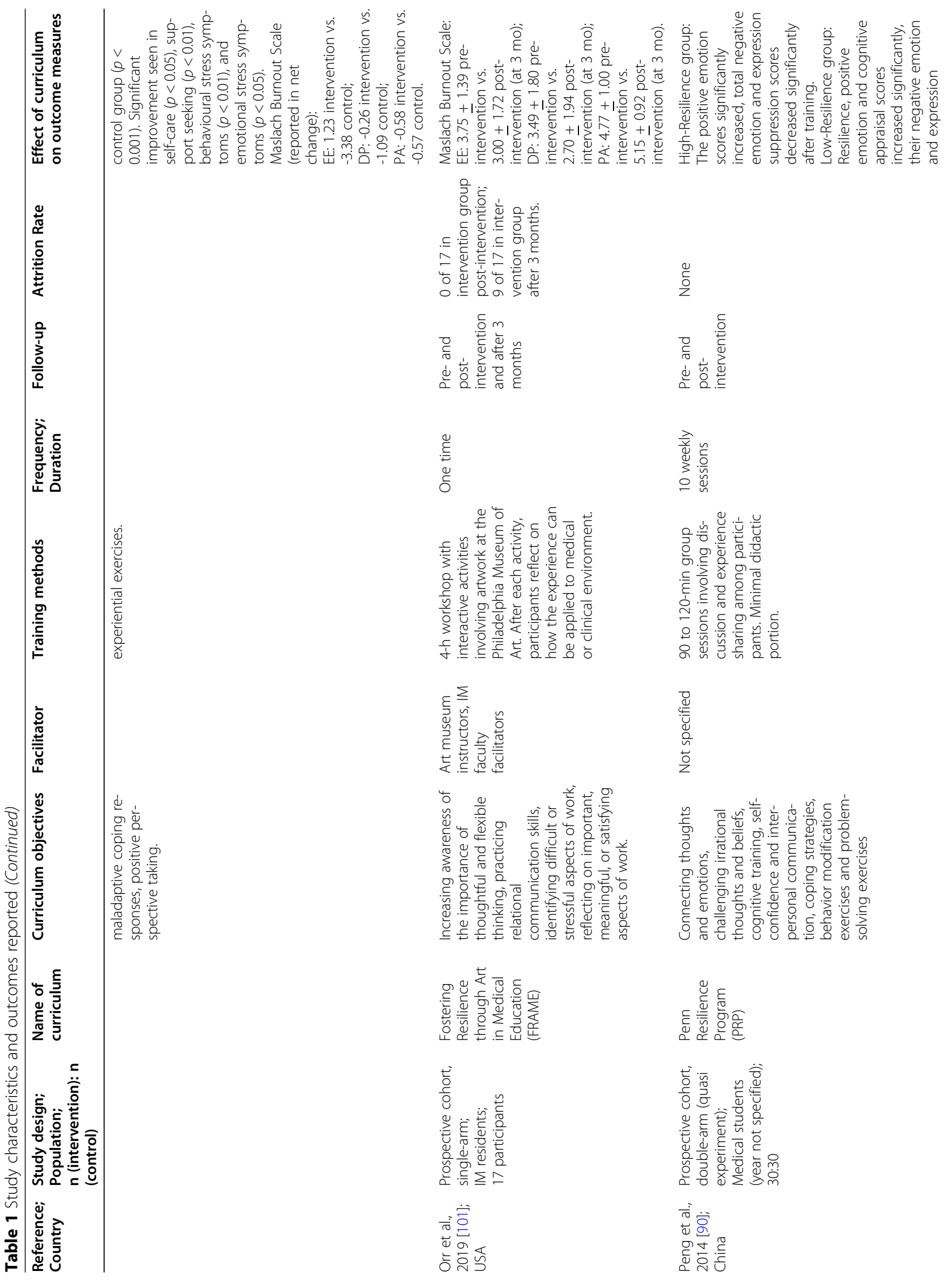




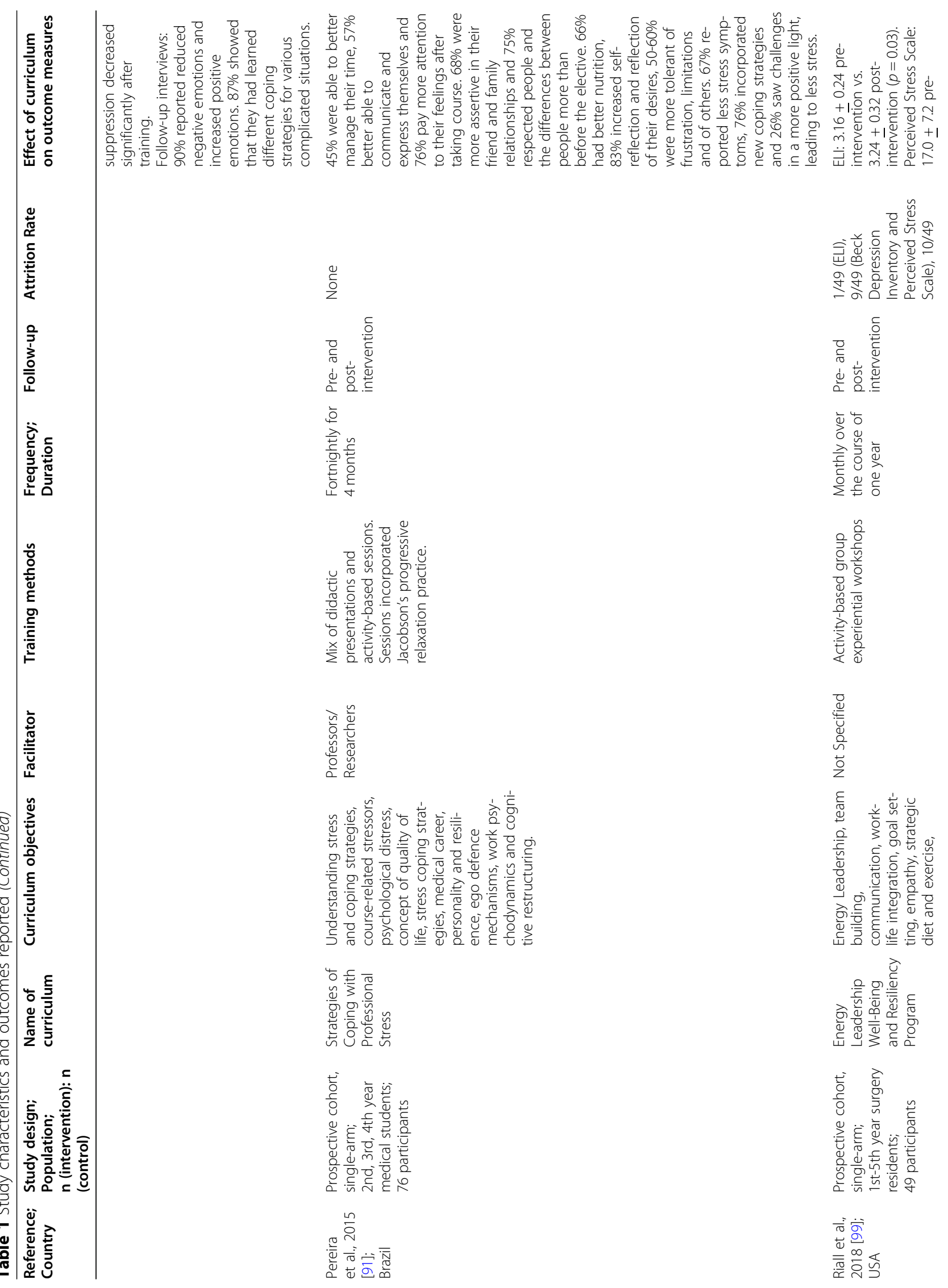




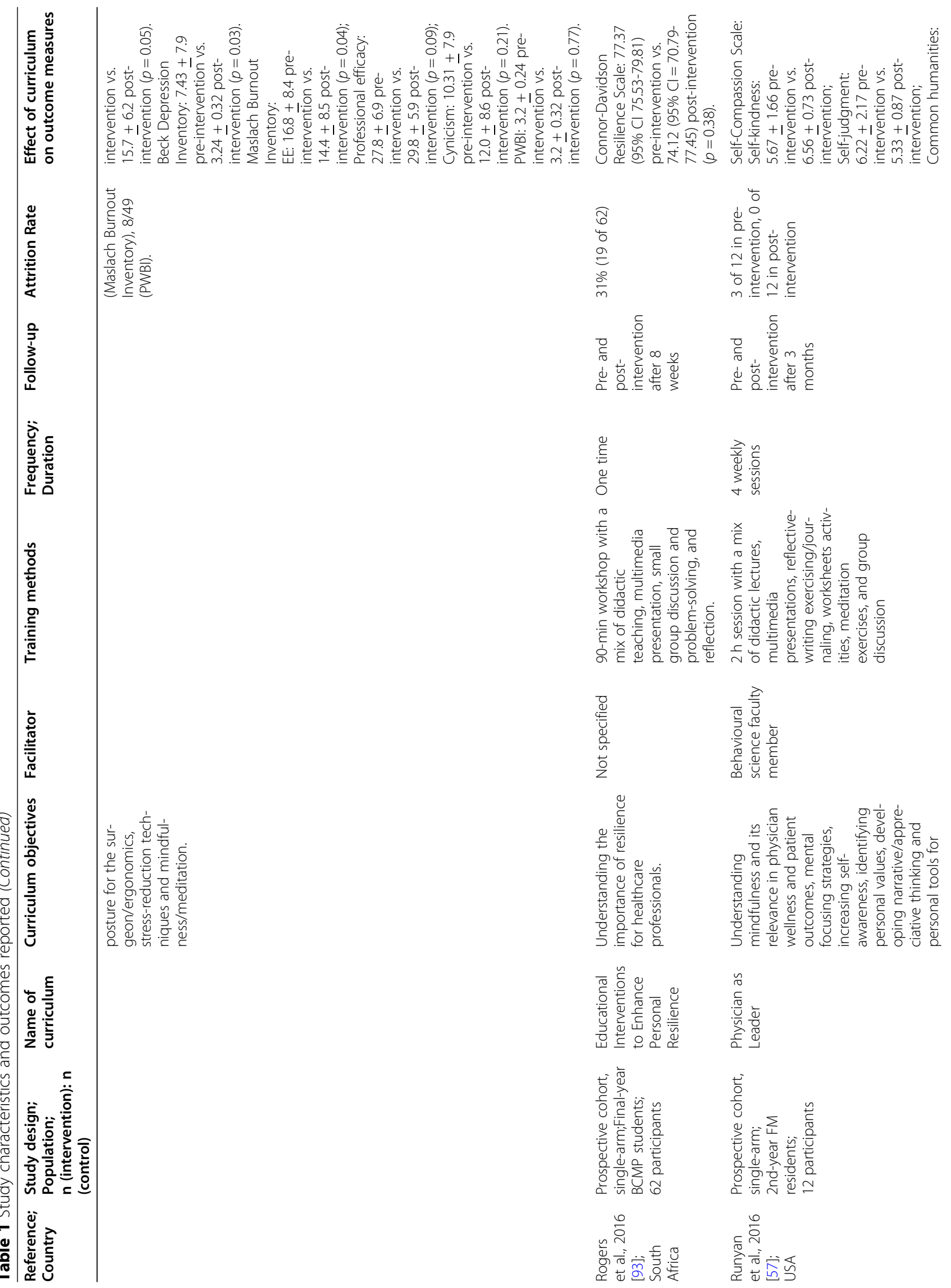




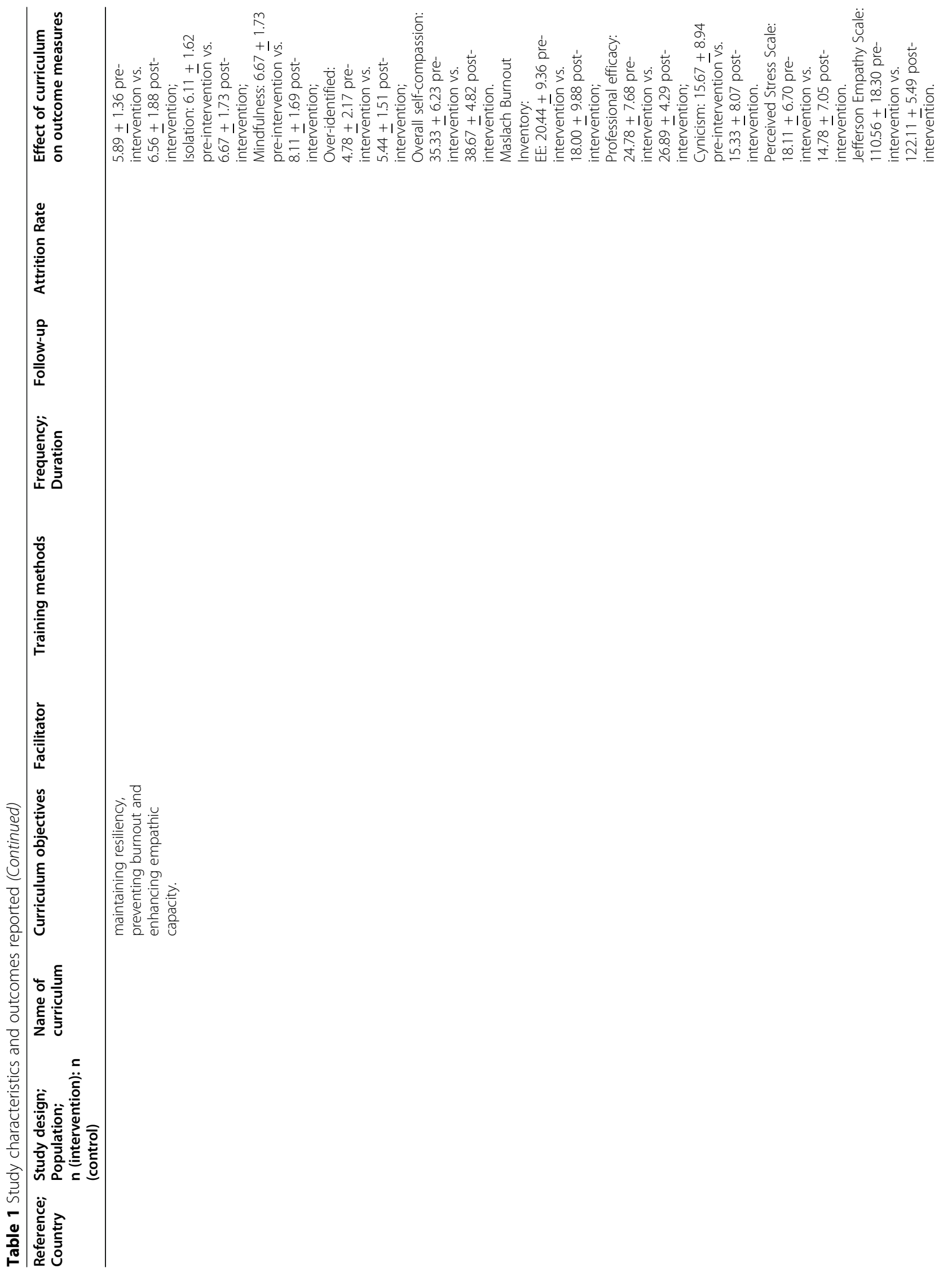




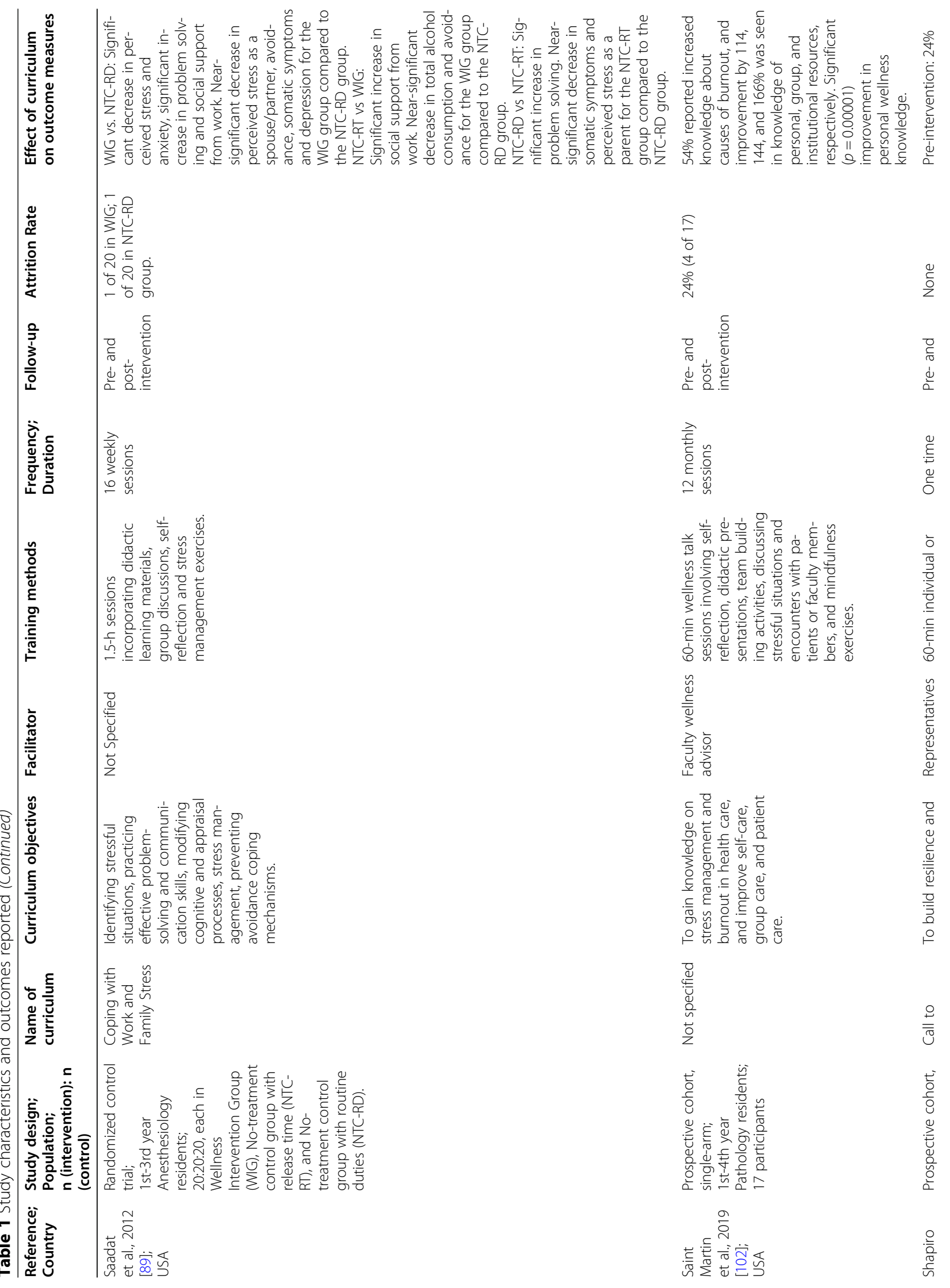




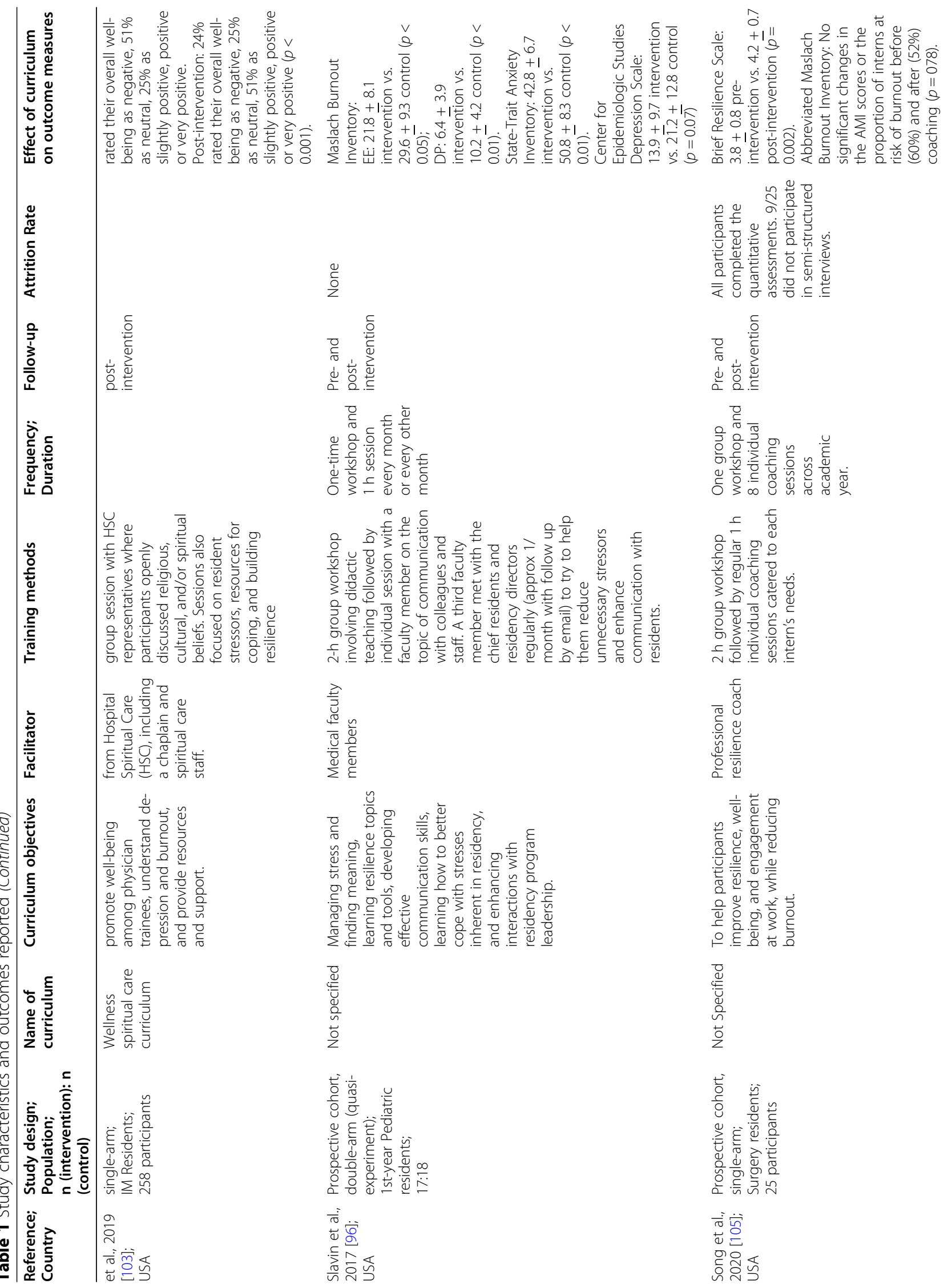




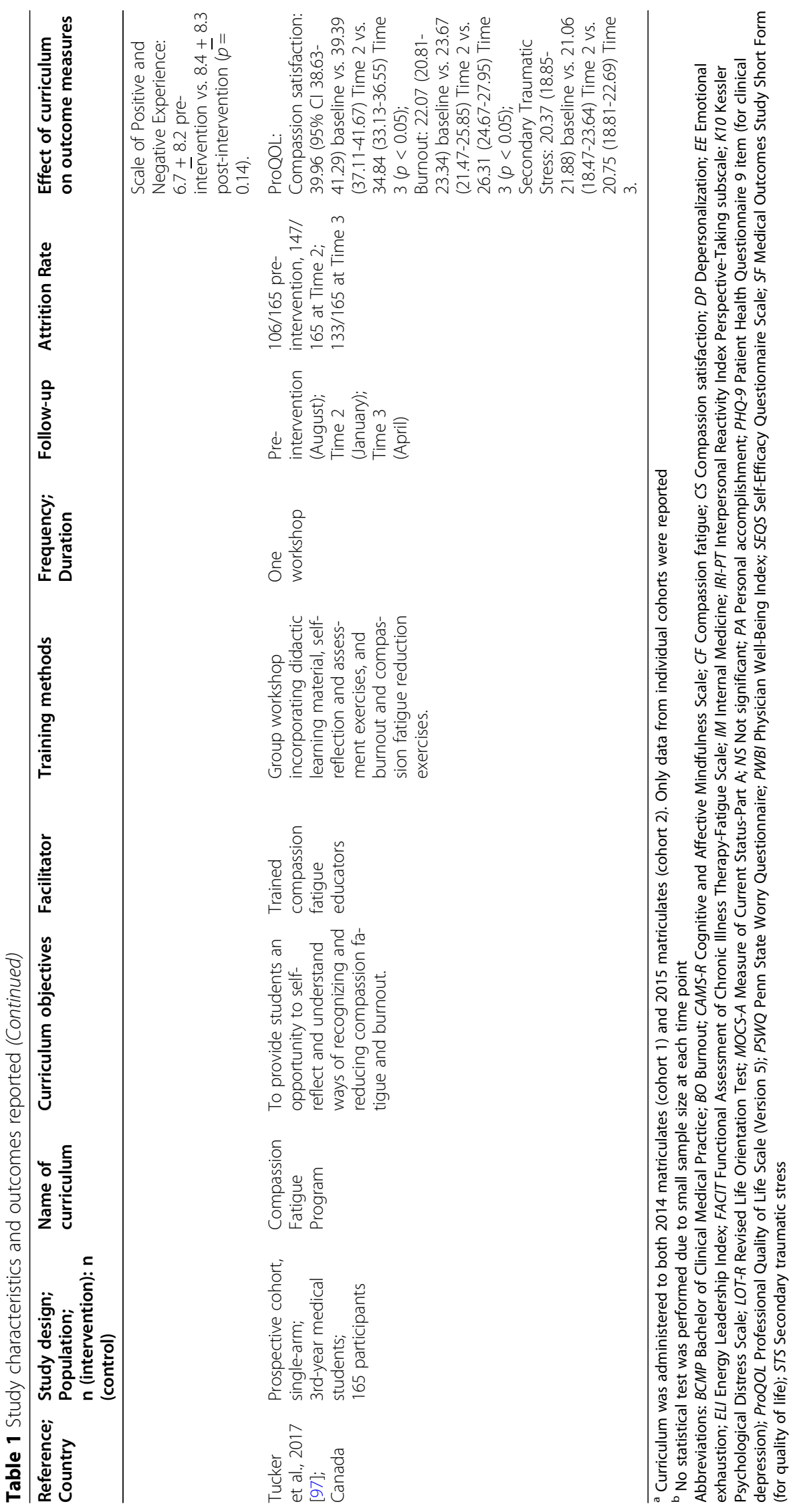




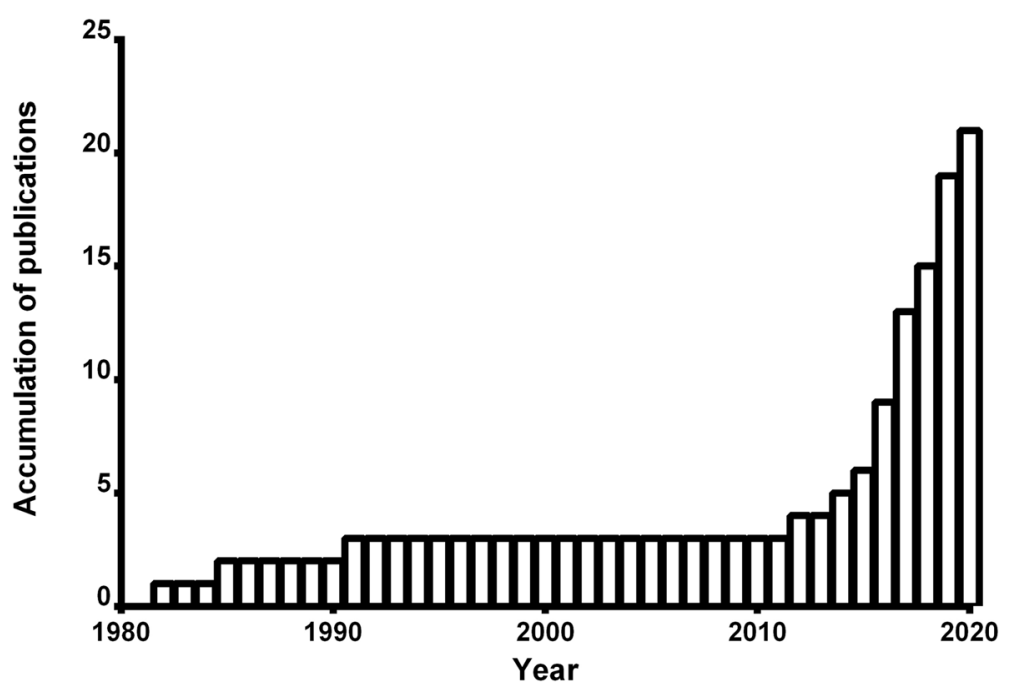

Fig. 2 Number of accumulated publications over time

participants. Sixty-two percent $(13 / 21, n=778)$ implemented the curricula in a PGME setting. Of the studies that tested their curricula among residents, 5 were in internal medicine, 2 in surgery, 2 in family medicine, 2 in pediatrics, 1 in psychiatry, 1 in anesthesiology, 1 in pathology, and 1 in internship. Geographic distribution of the included studies was heavily concentrated in the United States $(n=16)$, with only 1 study in Canada, China, Brazil, South Africa and Australia, respectively.

\section{Training methods}

Considerable heterogeneity existed in the duration and frequency of resilience curricula and ranged from a 1-h single session to 1.5 -h weekly sessions for 16 consecutive weeks. $76 \%(n=16)$ of the studies implemented inperson training over at least 4 sessions. The majority of studies incorporated more than one component to facilitate resilience, including didactic teaching sessions $(n=$ 16) and skill-building exercises around the themes of mindfulness, relaxation, and stress reduction $(n=16)$. Of the 5 resilience curricula that were based on previously developed training models, 2 used the Stress Management and Resilience Training Program [95, 98], and 3 used the Penn Resilience Program [90], Coping with Work and Family Stress Intervention [89], and Energy Leadership Well-Being and Resiliency Program [99], respectively. Two studies implemented non-conventional, outside-of-classroom curricular design. In a study by Shapiro et al. (2019), the authors sought to promote emotional well-being and resiliency in graduate medical trainees through individualized or group spiritual care sessions wherein residents were given an opportunity to openly discuss their religious, cultural, and spiritual beliefs with Hospital Spiritual Care representatives. In another study by Orr et al. (2019), senior internal medicine residents participated in a single, 4-h workshop at the Philadelphia Museum of Art that focused on the medical humanities and artful thinking exercises to enhance resilience and ameliorate burnout. There was a considerable variety in the background of instructors which included residents, psychologists, social workers, chaplain, and staff physicians. Only two studies were facilitated by trained resilience instructors $[97,105]$.

\section{Outcome measures}

All studies relied on self-report questionnaires to assess curricular outcomes. The majority of studies evaluated the effects of the resilience curriculum using previously validated outcome instruments $(90.5 \%, 19 / 21)$, while 2 studies used a new or non-proprietary measurement tool [91, 102]. Most commonly used outcome measure was Maslach Burnout Scale (9/21), followed by ConnorDavidson Resilience Scale (4/21), Perceived Stress Scale (4/21), Professional Quality of Life Scale (3/21), and Spielberger Trait Anxiety Inventory (3/21). Eleven studies assessed the effects of the curriculum immediately pre- and post-intervention with no follow-up. Only 4 studies reported long-term effects of their curriculum (i.e., after at least 12 months of delivering the curriculum).

As shown in Table 1, there was considerable heterogeneity in the efficacy of resilience curricula. While most studies suggested a modest improvement in their efficacy measures upon the completion of resilience curricula, a few studies reported no significant impact, and in some, even worsening of resilience and associated measures. Interestingly, despite positive feedback from students who also supported continuation of the resilience program, Bird et al. (2017) reported that both resilience and burnout measures significantly worsened post- 
intervention. Similarly, Chaukos et al. (2018) reported significant worsening of the EE and DP scores in the Maslach Burnout Inventory after administration of resilience curriculum and Dyrbye et al. (2017) reported an increasing trend of burnout on the Maslach Burnout Inventory in study participants. Due to the mixed and inconsistent outcome measures reported in the included studies, the overall benefit (or harm) of resilience curricula cannot be determined.

\section{Feasibility and acceptability}

Participant satisfaction and acceptability of resilience curricula were reported in 12 of the 21 studies [87, 88, 91, 94, 95, 97-99, 101, 103-105]. Participants reported generally positive experience and acceptability of resilience curricula, however, the methodology of program assessment was largely heterogeneous and unsystematic.

In Pereira et al. (2015), 90\% of the participants reported that the course was helpful, and $76.3 \%$ indicated that they had incorporated new coping mechanisms into daily life as a result of the training. Similarly, in Chaukos et al. (2018), 87\% considered the resilience skills taught during training to be helpful. While some participants suggested that resilience training should be part of mandatory curricula [91], others found it counterproductive and unnecessary [95].

In terms of curriculum duration, some participants indicated that resilience curricula should be implemented across all years of residency, as resilience skills and techniques may be more instrumental in later years with added stressors and clinical responsibilities [105]. Similarly, $75 \%$ of participants stated that the resilience curricula should continue in the following year [94]. 19\% reported that training sessions were too short in duration and infrequent [98]. Need for greater availability of resilience training and resources was noted by Tucker et al. (2017) as the authors quote responses from two participants: "I almost wish that there were more opportunities to talk about burnout during the year. I would have really appreciated that." and "Back in 1st and 2 nd year the student affairs office had these wellness checks. I wish we had that in third year because we can all sign up for one but a lot of us are too busy to even think of it."

Participants appreciated protected time for resilience curricula and having a safe space to discuss resilience topics: "[T] here was something special about having an opportunity to sort of vent about my experiences... I don't feel like there's anyone else in the program that I'd be willing to reveal that stuff to. And talking to each other is helpful, but it doesn't have that degree of separation" [105]. Similar trend of having a venue to openly discuss personal issues with peers was repeated in Pereira et al. (2015), Bird et al. (2017) and Tucker et al. (2017) as the participants felt the resilience curriculum provided a unique opportunity to build a community of shared experiences that allowed validation of participants' worries and hardships. Understandably, $8 \%$ reported feeling vulnerable in sessions [98].

\section{Risk of bias assessment}

To assess potential risk of bias, we used the ROBINS-I scoring system to evaluate bias across seven domains (Additional file 3): confounding bias, selection bias, classification bias, intervention bias, attrition bias, measurement bias, and reporting bias. Overall, the risk of bias was moderate for all studies, which, in light of the nonrandomized nature of the studies and reliance on selfreporting measures, is understandable. While confounding bias and classification bias were deemed to be low in most of the included studies, selection bias was moderate in $9(43 \%)$ and measurement bias was moderate in all $(100 \%)$ studies. Due to the high attrition rate in most studies, bias due to missing data was low in 6 studies (29\%), moderate in 12 studies (57\%), and unknown in 3 studies (14\%). To date, there have been only two randomized controlled trials that assessed the curricular efficacy of a resilience program, however these studies were assessed with the same bias scoring system to maintain coherence with other included studies.

\section{Discussion}

In response to the growing awareness of the high risk of burnout and stress in health professionals, there has been growing interest in resilience in medical education over the past decade. While physicians as a group have significantly higher resilience than the general public [106], efforts to overcome systemic challenges in the clinical care environment are truly needed to curve physician burnout and foster well-being. To the best of our knowledge, this is the first systematic review to provide a comprehensive summary of published literature on the effect of resilience curricula in undergraduate and postgraduate medical education. Our results highlight the considerable heterogeneity in content, delivery, and outcomes of resilience curricula implemented to date in medical education. In particular, in spite of the modest improvement in resilience and well-being reported in many studies, a number of other studies [94, 95, 98] suggested that the implementation of the program resulted in detrimental effects to the participants' overall resilience. Notably, all three studies delivered their curricula over 6 months to a year, and thus their negative results may have been confounded by an interaction between trainees' resilience level and the progression or level of training. True to the significant heterogeneity seen in the resilience curricula in this review, currently no standardized, efficacy-proven resilience curriculum exists around the world that could be implemented by 
undergraduate and postgraduate medical programs. This present review clearly calls for the development of more systematic and evidence-based programs and resources which are desperately needed in the fight to reduce physician stress and burnout.

The strengths of this review include its systematic methodology; prospectively registered with a clearly defined objective, comprehensive and detailed search strategy, and the screening of each study's risk of bias and methodological rigor. In spite of this, the findings of the present review should be interpreted in light of several limitations. Firstly, all included studies had moderate risk of bias according to the ROBINS-I tool for assessing the risk of bias in nonrandomized studies. Therefore, the results presented in this review must be interpreted in light of these potential biases. Secondly, most of the included studies did not assess long-term resilience outcomes of resilience programs. As such, it remains unclear whether the resilience curricula successfully fostered lasting resilience in medical students and residents, or if the reported benefits were transient. As previous research has shown resilience is an acquired skill and thought pattern that requires continual practice to achieve mastery, an assessment methodology that incorporates long-term follow-up on curriculum participants should be considered in future studies. In parallel, resilience curricula may result in long-term resilience building by providing strategies that trainees can then choose to continuously implement throughout their training and future practice. By providing appropriate tools, long-term sustainable resilience may be achievable. Next, all studies relied on self-reporting psychometric instruments and, as such, it is unclear whether the reported changes in the indices of resilience curricula have meaningful objective implications for personal and professional development. Furthermore, although an increasing number of resilience curricula are being offered throughout UGME and PGME, the results of this review demonstrate vast heterogeneity of curricular methods, ranging from one-time didactic lectures to a mix of lecture-based and practical sessions over many months. Consequently, a standardized approach to resilience curricula development does not currently exist. However, the lack of a common foundation between resilience programs is not surprising given the early nature of resilience-centered initiatives. Additionally, the diversity of medical trainees and trainee stressors may necessitate a resilience curriculum tailored specifically to their unique beliefs, values, and stressors. The broad range of curricula makes inter-institutional comparison difficult. More research is necessary to determine whether a standardized approach to resilience is feasible and beneficial, or if medical programs should develop unique curricula adapted to their trainees.

Despite these limitations, this systematic review provides a guidance for future resilience curricula development and related studies. Future research on resilience in medical education should include clear operationalization of resilience and curricular components to enable reproducibility and accurate comparison of outcomes. In addition, it would be beneficial to compare conventional in-class delivery of resilience curricula, the most common mode of delivery among the included studies, with other educational methods such as online learning and electronic interventions (e.g., smartphone apps) to explore their efficacy in different populations and contexts. Future research must assess the effect of varying duration and frequency of resilience curricula on participants' resilience and personal wellness; thereby elucidating the optimal curriculum delivery timeline. Lastly, future investigations must incorporate rigorous and robust research methodology to accurately determine the true effects of resilience training. A recent systematic review revealed that there is currently no gold standard outcome measure of resilience, thus rendering it difficult to assess criterion validity of various measures [107]. Further research should aim to develop a common instrument for objective resilience measurement.

The development and implementation of resilience education is a new phenomenon highlighted by an upstroke in publications during the past decade. Following recommendations by the ACGME in the United States and CanMEDS in Canada to improve trainee well-being, UGME and PGME programs in Canada and the US began to develop their own curricula to better support their trainees. Although medical trainee burnout has been extensively reported internationally, the implementation of resilience curricula has remained mostly limited to the US. Results of these preliminary studies can help to inform resilience education worldwide.

The benefits of resilience curricula are relevant to medical education, and at large, the medical workforce in today's society. Longitudinal studies have responded to the growing concern of the highly prevalent burnout and stress in the medical field and have demonstrated the predictive value of self-report resilience scales on future mental health problems in their career [108]. Yet, there remains hesitancy and lack of enthusiasm for routine provision of resilience training in medicine, where the belief that "doctors are invincible" remains fixed [109]. It is time to address the stigma, recognize vulnerabilities and push for cultural change. 


\section{Supplementary Information}

The online version contains supplementary material available at https://doi. org/10.1186/s12909-021-02495-0.

Additional file 1: PRISMA checklist.

Additional file 2: Search strategy.

Additional file 3: ROBINS-I risk of bias assessment.

\section{Acknowledgements}

Not applicable.

\section{Authors' contributions}

$\mathrm{CS}, \mathrm{MC}, \mathrm{TB}$, and $\mathrm{KH}$ conceived of the review idea. KF designed and performed searches in databases. CS and MC screened for eligible articles, extracted data, and wrote the manuscript. KH supervised the project. All authors provided critical feedback and have read and approved the final manuscript.

\section{Author's information}

C. Seo is a medical student at the University of Ottawa, Canada. M. Corrado is a medical student at the University of Ottawa, Canada. K. Fournier is a health sciences librarian at the University of Ottawa, Canada. T. Bailey is a family medicine resident at McMaster University, Canada. K. Haykal is a family physician and the Assistant Dean of Student Affairs at the Faculty of Medicine, University of Ottawa.

\section{Funding}

None.

\section{Availability of data and materials}

All data generated or analyzed during this study are included in this published article and its supplementary information files.

\section{Ethics approval and consent to participate}

Not applicable.

\section{Consent for publication}

Not applicable.

\section{Competing interests}

The authors declare that they have no competing interests.

\section{Author details}

${ }^{1}$ Department of Family Medicine, Faculty of Medicine, University of Ottawa, 451 Ch. Smyth Rd. (2024), Ottawa, ON K1H 8M5, Canada. ${ }^{2}$ Health Sciences Library, University of Ottawa, Ottawa, ON K1H 8M5, Canada. ${ }^{3}$ Department of Family Medicine, Faculty of Medicine, McMaster University, Hamilton, ON L8P 1 H6, Canada.

Received: 10 October 2020 Accepted: 4 January 2021

Published online: 01 February 2021

\section{References}

1. Raj KS. Well-being in residency: a systematic review. J Grad Med Educ. 2016; 8(5):674-84.

2. Cheng JW, Wagner H, Hernandez BC, Hu BR, Ko EY, Ruckle HC. Stressors and coping mechanisms related to burnout within urology. Urology. 2020;139: 27-36.

3. Seemann NM, Karanicolas PJ, Guttman MP, et al. Compassion fatigue in surgical trainees. J Surg Educ. 2019;76(5):1211-22.

4. Benson J, Magraith K. Compassion fatigue and burnout: the role of Balint groups. Aust Fam Physician. 2005;34(6):497-8.

5. Showalter SE. Compassion fatigue: what is it? Why does it matter? Recognizing the symptoms, acknowledging the impact, developing the tools to prevent compassion fatigue, and strengthen the professional already suffering from the effects. Am J Hosp Palliat Care. 2010;27(4):239-42.

6. Baste VS, Gadkari JV. Study of stress, self-esteem and depression in medical students and effect of music on perceived stress. Indian J Physiol Pharmacol. 2014;58(3):298-301.
7. Earle L, Kelly L. Coping strategies, depression, and anxiety among Ontario family medicine residents. Can Fam Physician. 2005:51:242-3.

8. Esan O, Esan A, Folasire A, Oluwajulugbe P. Mental health and wellbeing of medical students in Nigeria: a systematic review. Int Rev Psychiatry. 2019; 31(7-8):661-72.

9. Farrell SM, Moir F, Molodynski A, Bhugra D. Psychological wellbeing, burnout and substance use amongst medical students in New Zealand. Int Rev Psychiatry. 2019;31(7-8):630-636.

10. Farrell SM, Kadhum M, Lewis T, Singh G, Penzenstadler L, Molodynski A. Wellbeing and burnout amongst medical students in England. Int Rev Psychiatry. 2019;31(7-8):579-83.

11. Shi M, Liu L, Wang $Z Y$, Wang $L$. Prevalence of depressive symptoms and its correlations with positive psychological variables among Chinese medical students: an exploratory cross-sectional study. BMC Psychiatry. 2016;16:3.

12. Lee $\mathrm{KH}$, Ko Y, Kang KH, Lee HK, Kang J, Hur Y. Mental health and coping strategies among medical students. Korean J Med Educ. 2012;24(1):55-63.

13. Voltmer E, Rosta J, Aasland OG, Spahn C. Study-related health and behavior patterns of medical students: a longitudinal study. Med Teach. 2010;32(10): e422-8.

14. Ayala EE, Winseman JS, Johnsen RD, HRC M. U.S. medical students who engage in self-care report less stress and higher quality of life. BMC Med Educ. 2018;18(1):189

15. Lin YK, Lin C-D, Lin BY-J, Chen D-Y. Medical students' resilience: a protective role on stress and quality of life in clerkship. BMC Med Educ. 2019;19(1):473.

16. Messina G, Quercioli C, Troiano G, et al. Italian medical students quality of life: years 2005-2015. Ann lg. 2016;28(4):245-51.

17. Pariwatcharakul P, Ratta-Apha W, Sumalrot T, Wankaew J, Sitdhiraksa N Depression, quality of life and coping style among Thai doctors before their first year of residency training. Postgrad Med J. 2020;96(1136):321-4.

18. Tempski P, Bellodi PL, Paro HBMS, Enns SC, Martins MA, Schraiber LB. What do medical students think about their quality of life? A qualitative study. BMC Med Educ. 2012:12:106.

19. Tempski P, Santos IS, Mayer FB, et al. Relationship among medical student resilience, educational environment and quality of life. PLoS One. 2015:\#(6): e0131535.

20. West CP, Shanafelt TD, Kolars JC. Quality of life, burnout, educational debt, and medical knowledge among internal medicine residents. JAMA. 2011; 306(9):952-60.

21. Ahmady S, Khajeali N, Sharifi F, Mirmoghtadaei ZS. Factors related to academic failure in preclinical medical education: a systematic review. J Adv Med Educ Prof. 2019;7(2):74-85.

22. Kumar M, Sharma S, Gupta S, Vaish S, Misra R. Effect of stress on academic performance in medical students--a cross sectional study. Indian J Physiol Pharmacol. 2014:58(1):81-6.

23. Newbury-Birch D, Walshaw D, Kamali F. Drink and drugs: from medical students to doctors. Drug Alcohol Depend. 2001;64(3):265-70.

24. Croen LG, Woesner M, Herman M, Reichgott M. A longitudinal study of substance use and abuse in a single class of medical students. Acad Med. 1997;72(5):376-81.

25. Steiner-Hofbauer $V$, Holzinger A. How to cope with the challenges of medical education? Stress, depression, and coping in undergraduate medical students. Acad Psychiatry. 2020;44(4):380-7.

26. Thomas MR, Dyrbye LN, Huntington $J$, et al. How do distress and wellbeing relate to medical student empathy? A multicenter study. J Gen Intern Med. 2007:22(2):177-83.

27. McFarland DC, Roth A. Resilience of internal medicine house staff and its association with distress and empathy in an oncology setting. Psychooncology. 2017;26(10):1519-25.

28. Dyrbye LN, Massie FS Jr, Eacker A, et al. Relationship between burnout and professional conduct and attitudes among US medical students. JAMA. 2010;304(11):1173-80.

29. Dyrbye LN, Thomas MR, Massie FS, et al. Burnout and suicidal ideation among U.S. medical students. Ann Intern Med. 2008;149(5):334-41.

30. Bailey E, Robinson J, McGorry P. Depression and suicide among medical practitioners in Australia. Intern Med J. 2018;48(3):254-8.

31. Rodrigues $H$, Cobucci $R$, Oliveira $A$, et al. Burnout syndrome among medical residents: a systematic review and meta-analysis. PLoS One. 2018;13(11): e0206840.

32. Rothenberger DA. Physician burnout and well-being: a systematic review and framework for action. Dis Colon Rectum. 2017;60(6):567-76. 
33. MacLean L, Booza J, Balon R. The impact of medical school on student mental health. Acad Psychiatry. 2016;40:89-91.

34. Chan MK, Chew QH, Sim K. Burnout and associated factors in psychiatry residents: a systematic review. J Int Assoc Med Sci Educ. 2019;10:149-60

35. Frajerman A, Morvan Y, Krebs M-O, Gorwood P, Chaumette B. Burnout in medical students before residency: a systematic review and meta-analysis. Eur Psychiatry. 2019;55:36-42.

36. Pacheco JP, Giacomin HT, Tam WW, et al. Mental health problems among medical students in Brazil: a systematic review and meta-analysis. Braz J Psychiatry. 2017;39(4):369-78.

37. Mao Y, Zhang N, Liu J, Zhu B, He R, Wang X. A systematic review of depression and anxiety in medical students in China. BMC Med Educ. 2019; 19(1):327.

38. Erschens R, Keifenheim KE, Herrmann-Werner A, et al. Professional burnout among medical students: systematic literature review and meta-analysis. Med Teach. 2019;41(2):172-83.

39. Afana A, Ghannam J, Ho EY, Al-Khal A, Al-Arab B, Bylund CL. Burnout and sources of stress among medical residents at Hamad Medical Corporation, Qatar. East Mediterr Health J. 2017;23(1):40-5.

40. Kealy D, Halli P, Ogrodniczuk JS, Hadjipavlou G. Burnout among Canadian psychiatry residents: a national survey. Can J Psychiatry. 2016;61(11):732-6.

41. Doolittle BR, Windish DM. Correlation of burnout syndrome with specific coping strategies, behaviors, and spiritual attitudes among interns at Yale University, New Haven, USA. J Educ Eval Health Prof. 2015;12:41.

42. Galam E, Vauloup Soupault C, Bunge L, Buffel du Vaure C, Boujut E, Jaury P. "Intern life": a longitudinal study of burnout, empathy, and coping strategies used by French GPs in training. BJGP Open. 2017;1 (2):bjgpopen17X100773.

43. Nimmawitt N, Wannarit K, Pariwatcharakul P. Thai psychiatrists and burnout: a national survey. PLoS One. 2020;15(4):e0230204.

44. Sargent MC, Sotile W, Sotile MO, et al. Stress and coping among orthopaedic surgery residents and faculty. J Bone Joint Surg Am. 86:1579-86.

45. Sreelatha P, Premlal L, Ryali VSSR. Burnout and coping strategies among residents of a private medical college in South India: a cross-sectional study. Ind Psychiatry J. 2018;27(2):213-8.

46. Youssef FF. Medical student stress, burnout and depression in Trinidad and Tobago. Acad Psychiatry. 2016;40(1):69-75

47. Zis $\mathrm{P}$, Artemiadis AK, Lykouri $\mathrm{M}$, et al. Residency training: determinants of burnout of neurology trainees in Attica, Greece. Neurology. 2015;85(11):e81-4.

48. Jennings ML, Slavin SJ. Resident wellness matters: optimizing resident education and wellness through the learning environment. Acad Med. 2015;90(9):1246-50.

49. Sargent MC, Sotile W, Sotile MO, et al. Managing stress in the orthopaedic family: avoiding burnout, achieving resilience. J Bone Joint Surg Am. 2011; 93(8):e40.

50. Maslach C, Leiter MP. Understanding the burnout experience: recent research and its implications for psychiatry. World Psychiatry. 2016;15(2): 103-11.

51. Berwick DM, Nolan TW, Whittington J. The triple aim: care, health, and cost. Health Aff. 2008;27(3):759-69.

52. Bodenheimer T, Sinsky C. From triple to quadruple aim: care of the patient requires care of the provider. Ann Fam Med. 2014;12(6):573-6.

53. 2015 ACGME Symposium on Physician Well-Being - Summary And Proposal to the ACGME Board of Directors. Accreditation Council for Graduate Medical Education. Retrieved on September 29, 2020. https://www.acgme. org/What-We-Do/Initiatives/Physician-Well-Being/2015-ACGMESymposiumon-Physician-Well-Being.

54. Summary of changes to ACGME common program requirements. Section VI.C. Well-being. Accreditation Council for Graduate Medical Education. Retrieved on September 29, 2020. https://www.acgme.org/What-We-Do/ Accreditation/Common-Program-Requirements/Summary-of-ProposedChangesto-ACGME-Common-Program-Requirements-Section-VI.

55. Frank JR, Snell L, Sherbino J. CanMEDS 2015 Physician Competency Framework. Royal College of Physicians and Surgeons of CA; 2015. CanMEDS. Retrieved on September 29 from http://canmeds.royalcollege.ca/ en/framework

56. Eckleberry-Hunt J, Van Dyke A, Lick D, Tucciarone J. Changing the conversation from burnout to wellness: physician well-being in residency training programs. J Grad Med Educ. 2009; 1(2):225-30

57. Runyan C, Savageau JA, Potts S, Weinreb L. Impact of a family medicine resident wellness curriculum: a feasibility study. Med Educ Online. 2016;21: 30648.
58. Hassed C, de Lisle S, Sullivan G, Pier C. Enhancing the health of medical students: outcomes of an integrated mindfulness and lifestyle program. Adv Health Sci Educ Theory Pract. 2009;14(3):387-98.

59. Rosenzweig S, Reibel DK, Greeson JM, Brainard GC, Hojat M. Mindfulnessbased stress reduction lowers psychological distress in medical students. Teach Learn Med. 2003;15(2):88-92.

60. Warnecke E, Quinn S, Ogden K, Towle N, Nelson MR. A randomised controlled trial of the effects of mindfulness practice on medical student stress levels. Med Educ. 2011;45(4):381-8.

61. Simard A-A, Henry M. Impact of a short yoga intervention on medical students' health: a pilot study. Med Teach. 2009;31(10):950-2.

62. Cocchiara RA, Peruzzo M, Mannocci A, et al. The use of yoga to manage stress and burnout in healthcare workers: a systematic review. J Clin Med Res. 2019;8(3):284.

63. Whitehouse WG, Dinges DF, Orne EC, et al. Psychosocial and immune effects of self-hypnosis training for stress management throughout the first semester of medical school. Psychosom Med. 1996;58(3):249-63.

64. Redwood SK, Pollak MH. Student-led stress management program for firstyear medical students. Teach Learn Med. 2007;19(1):42-6.

65. Kiessling C, Schubert B, Scheffner D, Burger W. First year medical students' perceptions of stress and support: a comparison between reformed and traditional track curricula. Med Educ. 2004;38(5):504-9.

66. Bloodgood RA, Short JG, Jackson JM, Martindale JR. A change to pass/fail grading in the first two years at one medical school results in improved psychological well-being. Acad Med. 2009;84(5):655-62.

67. Reed DA, Shanafelt TD, Satele DW, et al. Relationship of pass/fail grading and curriculum structure with well-being among preclinical medical students: a multi-institutional study. Acad Med. 2011;86(11):1367-73.

68. Lincoln M, Adamson BJ, Covic T. Teaching time and organizational management skills to first year health science students: does training make a difference? J Furth High Educ. 2004;28(3):261-76.

69. DasGupta S, Charon R. Personal illness narratives: using reflective writing to teach empathy. Acad Med. 2004;79(4):351-6.

70. Austenfeld JL, Paolo AM, Stanton AL. Effects of writing about emotions versus goals on psychological and physical health among third-year medical students. J Pers. 2006;74(1):267-86.

71. Holm M, Tyssen R, Stordal Kl, Haver B. Self-development groups reduce medical school stress: a controlled intervention study. BMC Med Educ. 2010;10:23.

72. Berger $\mathrm{L}$, Waidyaratne-Wijeratne $\mathrm{N}$. Where does resiliency fit into the residency training experience: a framework for understanding the relationship between wellness, burnout, and resiliency during residency training. Can Med Educ J. 2019;10(1):e20-7.

73. Shiralkar MT, Harris TB, Eddins-Folensbee FF, Coverdale JH. A systematic review of stress-management programs for medical students. Acad Psychiatry. 2013;37(3):158-64

74. Howe A, Smajdor A, Stöckl A. Towards an understanding of resilience and its relevance to medical training. Med Educ. 2012;46(4):349-56.

75. Epstein RM, Krasner MS. Physician resilience: what it means, why it matters, and how to promote it. Acad Med. 2013;88(3):301-3.

76. McKinley N, Karayiannis PN, Convie L, Clarke M, Kirk SJ, Campbell WJ. Resilience in medical doctors: a systematic review. Postgrad Med J. 2019; 95(1121):140-7.

77. Brennan J, McGrady A. Designing and implementing a resiliency program for family medicine residents. Int J Psychiatry Med. 2015;50(1):104-14.

78. Gerrard N, Kulig J, Nowatzki N. What doesn't kill you makes you stronger: determinants of stress resiliency in rural people of Saskatchewan, Canada. J Rural Health. 2004;20(1):59-66.

79. Walsh K. An economic argument for investment in physician resilience. Acad Med. 2013;88(9):1196.

80. Nedrow A, Steckler NA, Hardman J. Physician resilience and burnout: can you make the switch? Fam Pract Manag. 2013;20(1):25-30.

81. Shamseer L, Moher D, Clarke M, et al. Preferred reporting items for systematic review and meta-analysis protocols (PRISMA-P) 2015: elaboration and explanation. BMJ. 2015;350:97647.

82. Moher D, Liberati A, Tetzlaff J, Altman DG, PRISMA Group. Preferred reporting items for systematic reviews and meta-analyses: the PRISMA statement. PLoS Med. 2009;6(7):e1000097.

83. Higgins J, Thomas J. Cochrane Handbook for Systematic Reviews of Interventions. The Cochrane Collaboration; Updated August 2019.

84. Higgins JPT, Altman DG, Gøtzsche PC, et al. The Cochrane Collaboration's tool for assessing risk of bias in randomised trials. BMJ. 2011;343:d5928. 
85. Sterne JA, Hernán MA, Reeves BC, et al. ROBINS-1: a tool for assessing risk of bias in non-randomised studies of interventions. BMJ. 2016;355:14919.

86. Kelly JA, Bradlyn AS, Dubbert PM, St Lawrence JS. Stress management training in medical school. J Med Educ. 1982;57(2):91-9.

87. Holtzworth-Munroe A, Munroe MS, Smith RE. Effects of a stressmanagement training program on first- and second-year medical students. J Med Educ. 1985;60(5):417-9.

88. McCue JD, Sachs CL. A stress management workshop improves residents' coping skills. Arch Intern Med. 1991;151(11):2273-7.

89. Saadat H, Snow DL, Ottenheimer S, Dai F, Kain ZN. Wellness program for anesthesiology residents: a randomized, controlled trial. Acta Anaesthesiol Scand. 2012;56(9):1130-8.

90. Peng L, Li M, Zuo X, et al. Application of the Pennsylvania resilience training program on medical students. Pers Individ Dif. 2014;61-62:47-51.

91. Pereira MAD, Barbosa MA, de Rezende JC, Damiano RF. Medical student stress: an elective course as a possibility of help. BMC Res Notes. 2015;8:430.

92. Brennan J, McGrady A, Lynch DJ, Schaefer P, Whearty K. A stress management program for higher risk medical students: preliminary findings. Appl Psychophysiol Biofeedback. 2016;41(3):301-5.

93. Rogers D. Does a brief workshop change clinical associate students' resilience? Afr J Prim Health Care Fam Med. 2016;8(1):e1-4.

94. Bird A-N, Martinchek M, Pincavage AT. A curriculum to enhance resilience in internal medicine interns. J Grad Med Educ. 2017;9(5):600-4.

95. Dyrbye LN, Shanafelt TD, Werner L, Sood A, Satele D, Wolanskyj AP. The impact of a required longitudinal stress management and resilience training course for first-year medical students. J Gen Intern Med. 2017:32(12):1309-14.

96. Slavin S, Shoss M, Broom MA. A program to prevent burnout, depression, and anxiety in first-year pediatric residents. Acad Pediatr. 2017;17(4):456-8.

97. Tucker T, Bouvette M, Daly S, Grassau P. Finding the sweet spot: developing, implementing and evaluating a burn out and compassion fatigue intervention for third year medical trainees. Eval Program Plann. 2017;65:106-12.

98. Chaukos D, Chad-Friedman E, Mehta DH, et al. SMART-R: a prospective cohort study of a resilience curriculum for residents by residents. Acad Psychiatry. 2018;42(1):78-83.

99. Riall TS, Teiman J, Chang M, et al. Maintaining the fire but avoiding burnout: implementation and evaluation of a resident well-being program. J Am Coll Surg. 2018;226(4):369-79.

100. Brennan J, McGrady A, Tripi J, et al. Effects of a resiliency program on burnout and resiliency in family medicine residents. Int J Psychiatry Med. 2019;54(4-5):327-35.

101. Orr AR, Moghbeli N, Swain A, et al. The fostering resilience through art in medical education (FRAME) workshop: a partnership with the Philadelphia Museum of art. Adv Med Educ Pract. 2019;10:361-9.

102. Saint Martin MC, DeChristopher PJ, Sweeney RP. A strategy for wellness in a pathology residency program: enhancing chances of success during an epidemic of burnout. Acad Pathol. 2019:6:2374289519851233.

103. Shapiro RE, Vallejo MC, Sofka SH, Elmo RM, Anderson AH, Ferrari ND. Hospital spiritual care can complement graduate medical trainee wellbeing. Adv Med. 2019;2019:8749351.

104. Forbes M, Byrom L, van der Steenstraten I, Markwell A, Bretherton H, Kay M. Resilience on the run: an evaluation of a well-being programme for medical interns. Intern Med J. 2020;50(1):92-9.

105. Song Y, Swendiman RA, Shannon AB, et al. Can we coach resilience? An evaluation of professional resilience coaching as a well-being initiative for surgical interns. J Surg Educ. 2020;77(6):1481-9.

106. West C, Dyrbye LN, Sinsky M, et al. Resilience and burnout among physicians and the general US working population. JAMA Netw Open. 2020; 3(7):e209385. https://doi.org/10.1001/jamanetworkopen.2020.9385.

107. Windle G, Bennett KM, Noyes J. A methodological review of resilience measurement scales. Health Qual Life Outcomes. 2011;9:8.

108. Wild J, Smith KV, Thompson E, Béar F, Lommen MJJ, Ehlers A. A prospective study of pre-trauma risk factors for post-traumatic stress disorder and depression. Psychol Med. 2016;46(12):2571-82.

109. Henderson M, Brooks SK, Del Busso L, et al. Shame! Self-stigmatisation as an obstacle to sick doctors returning to work: a qualitative study. BMJ Open. 2012;2(5):e001776.

\section{Publisher's Note}

Springer Nature remains neutral with regard to jurisdictional claims in published maps and institutional affiliations.

Ready to submit your research? Choose BMC and benefit from:

- fast, convenient online submission

- thorough peer review by experienced researchers in your field

- rapid publication on acceptance

- support for research data, including large and complex data types

- gold Open Access which fosters wider collaboration and increased citations

- maximum visibility for your research: over $100 \mathrm{M}$ website views per year

At BMC, research is always in progress.

Learn more biomedcentral.com/submissions 